\title{
SUSTAINABILITY OF MINORITY CULTURE WHEN INTER-ETHNIC INTERACTION IS PROFITABLE
}

\author{
JOHN BUNCE*1,2,3 AND RICHARD MCELREATH ${ }^{1,2}$
}

1 AbStRaCt. Theoretical models suggest that cultural variation among ethnic groups can be maintained if inter-ethnic interaction is curtailed, or if groups are balanced in size and prestige. However, inter-ethnic interaction often takes place between members of groups that differ in size and prestige, as well as in power. We construct a model to examine the effects of ethnic asymmetries in inter-ethnic coordination benefits on changes in the frequency of cultural norms in a population comprising minority and majority ethnic groups. In the absence of other processes, conditions favourable for the sustainability of minority culture may be broadened through establishment of a territory with a border that is permeable to members of a dis-empowered minority group, but not to members of a powerful majority. We show how model predictions 1 can complement empirical studies of cultural change, and demonstrate the model's 12 relevance to our understanding of norm dynamics in an indigenous Amazonian pop13 ulation.

Keywords: cultural evolution, ethnicity, norms, minorities, territory, Amazonia

\footnotetext{
${ }^{1}$ Max Planck Institute for Evolutionary Anthropology, Deutscher Platz 6, 04103 Leipzig, Germany. ${ }^{2}$ Department of Anthropology, University of California, Davis, One Shields Ave, Davis CA 95616. ${ }^{3}$ Department of Anthropology, Indiana University, 701 E. Kirkwood Avenue, Bloomington, IN 47405

E-mail address: john_bunce@eva.mpg.de.
} 


\section{INTRODUCTION}

The extent of the evolved human capacity for and reliance on social learning is unique in nature. These characteristics are responsible for our species' unprecedented variation in behaviour and belief, our rapid rate of adaptation, and our immense success compared to other primates (Richerson and Boyd 2005). Social learning generates cultural variation - neighbouring groups of people often think and behave quite differently, owing partly to socially transmitted ideas and strategies. Such cultural variation is often adaptive - different norms, customs, and behaviour often fit local circumstances in ways that enhance human welfare. Cultural variation is often valued - groups of people sometimes identify with and seek to preserve their distinctive cultures and prevent their replacement by those of other groups with whom they interact (e.g., Kopenawa and Albert 2013, Nagel 1996). However, cultural variation can also be lost - individuals sometimes see benefits in cultural change and the adoption of certain out-group cultural characteristics (e.g., Aikman 2003, Baer 2004).

Therefore, from both the objective perspective of adaptation and the subjective perspective of preserving cultural traditions, evolutionary anthropology requires a robust theory of why cultural variation is sometimes maintained under intense inter-ethnic interaction, as well as why it is sometimes lost (Bunce and McElreath 2017). Here we use a dynamic model of interaction and cultural transmission to ask: When individuals act in their own short-term self-interest, under which conditions can cultural variation be sustained?

\subsection{Theoretical models of cultural change among groups. Several answers} to this question are suggested by recent mathematical models of cultural evolution. McElreath et al. (2003) show that stable groups with distinctive cultural norms and salient markers of group membership can evolve from a population without such structure if inter-individual interaction takes the form of coordination, interaction partners are chosen on the basis of arbitrary markers, and norms and markers tend to be acquired from locally successful individuals. Similarly, Boyd and Richerson (1987) show that marked groups characterized by distinctive cultural adaptations to the environment can evolve if ecology varies spatially, and people prefer to copy behaviour from successful individuals with markers like their own. In both of these models, once group-structure evolves in a population, inter-group interactions in the form of attempted coordination or cultural copying are invariably more costly to individuals on average than are such interactions with other group members. Thus, distinctive group-level cultural traits coincide with the evolution of mechanisms that effectively curtail inter-group interaction.

Considerable attention has been paid to models illustrating mechanisms by which the cultural traits of one group can replace those of another. For instance, Boyd and Richerson (1990) show that cultural traits yielding greater group-level benefits can spread when one group out-competes another through relative increases in its membership. Using a different mathematical approach, Gilpin et al. (2016) construct a model of competition between groups with distinctive internal cultural dynamics. The presence of one group is assumed to be invariably antagonistic to the growth of the other, and this is used to represent the demographic (and perhaps cultural) replacement of Neanderthals by modern humans. Modelling a mechanism first proposed 
by Henrich and Gil-White (2001), Boyd and Richerson (2002) demonstrate that cultural traits associated with successful or prestigious groups can spread through biased copying by people in less-prestigious groups. In a more recent model, Boyd and Richerson (2009) show that cultural traits associated with groups that attract migrants can become more common in a population as people are culturally assimilated into these attractive (e.g., successful or prestigious) groups, though this depends strongly on initial trait frequencies and migration rates. Thus, in these models, group-level cultural variation can be difficult to preserve if one group is less demographically successful or less subjectively prestigious than another.

In summary, current theoretical models of cultural change suggest that cultural traits typical of a particular group can often be maintained if: 1) inter-group interaction is costly and is thus curtailed; or 2) the group's membership grows at least as fast as that of other groups; and 3) the group is at least as subjectively prestigious as other groups. These conclusions are based on models that share a common feature: Interaction between individuals of culturally-distinct groups is never considered to be directly beneficial to those individuals. Rather, such interaction is invariably costly on average due to the risk of inter-group miscoordination (McElreath et al. 2003), is not explicitly modelled (Boyd and Richerson 1990, 2009, Gilpin et al. 2016), or consists only of cultural copying that has an indirect effect on individual payoffs after exposure to the environment (Boyd and Richerson 1987) or interaction with other group members (Boyd and Richerson 2002). In the real world, however, there are often immediate and substantial subjective benefits to peaceful interaction with someone from a different ethnic group or nation. As in the model of McElreath et al. (2003), cultural differences may make inter-ethnic interaction risky and prone to failure. However, if it is successful, the benefits are often greater than benefits obtained from interaction with co-ethnics, e.g., access to non-local resources, wages, labour, or technology (Bunce and McElreath 2017, Pisor and Gurven 2016, Sahlins 1994, Kopenawa and Albert 2013, Solway and Lee 1990). Such benefits are most likely the reason why individual-level inter-ethnic interaction, particularly exchange of resources, services, and information, is nearly universal in all known historical and ethnographic contexts (e.g., Wolf 1982, Solway and Lee 1990, Ross and Atkinson 2016). At present, we lack a theoretical understanding of cultural change in ethnically-structured populations where individual benefits from inter-ethnic interaction can potentially outweigh benefits from intra-ethnic interaction. Here we construct a formal model to explore cultural dynamics in such a context, focusing on minimal conditions for the preservation of cultural traits of minority ethnic groups.

1.2. Cultural norms and coordination. An important component of human culture comprises suites of beliefs about what constitutes appropriate behaviour in a given context. Such beliefs (or rules) are often crucial to an individual's selfidentification with, and ascription by others to, a particular ethnic group. We call such beliefs norms (Barth 1998; see also Bicchieri 2006). Norms play a particularly important role in inter-ethnic interactions because many such interactions have the form of coordination games. In a coordination game, all participants receive a higher (though not necessarily equal) payoff if they act in concordant rather than discordant manners (Bicchieri 2006). Consequently, players should seek to interact with others holding similar norms in the context of interaction. Many domains of social 
life, such as commerce, labour, healthcare, education, and marriage (Nave 2000) can be modelled as coordination games. Inter-ethnic interactions can be especially challenging because, initially, distributions of norms often differ among ethnic groups (Barth 1998), potentially frustrating attempts at coordination. Despite this, individuals often persist in such inter-ethnic interaction because, if coordination eventually becomes successful, substantial benefits may be realized (e.g., 18th century ChineseBritish commerce: Sahlins 1994). In such situations, people must weigh the greater payoff of inter-ethnic coordination against the greater risk of miscoordination.

1.3. A model of cultural norms and group asymmetries. This topic is sufficiently complex such that further empirical work and further theorizing can both contribute substantially to our understanding. Useful empirical work will address phenomena of theoretical importance, while still being open to the additional complexity and surprise of natural systems. Useful models must be unrealistically simple, so that they can shed light on the logic of particular hypotheses, while still being guided by empiricism.

Here we take up the modelling challenge. The model we present below addresses the dynamics of cultural norms in a group-structured population where minority and majority groups are culturally distinct, people voluntarily attempt coordination interactions, and individuals tend to adopt the norms of those who are successful in such interactions. We modify marginal payoffs so that inter-group coordination, if it can be achieved, is more profitable to individuals than is intra-group coordination. However, we allow such payoffs to differ between groups. We show that, given plausible asymmetries in inter-group coordination benefits (i.e., differential bargaining power), degrees of assortment during coordination, and rates of inter-group interaction, cultural norms of minority groups can be maintained if the minority occupies a territory that does not attract majority visitors, or that has a boundary which is permeable to minority, but not majority, members. These constitute theoretical sufficient conditions for the sustainability of minority cultural norms in the absence of other processes influencing cultural change. In the Discussion we show an application of model predictions to cultural change in an Amazonian population.

2.1. Population structure. Consider a population of arbitrarily large size. There exist two alternative cultural norms in the population, designated norm 1 and norm 2 , with $p_{1}$ representing the frequency of norm 1 and $p_{2}=1-p_{1}$. The population is completely divided into a minority group $\mathrm{S}$ (Small) and a majority group B (Big), such that group $\mathrm{S}$ has $d_{S}$ times the number of constituents of group B, and $d_{B}=1 / d_{S}$. In this model, $d_{S}$ is always $<1$. The frequency of norm 1 in group $\mathrm{S}$ is $p_{1 S}$, where the first subscript represents the norm and the second the group. Initially, norm 1 occurs at high frequency in group $\mathrm{S}$ (large $p_{1 S}$, small $p_{2 S}$ ), while norm 2 occurs at high frequency in group $\mathrm{B}$ (large $p_{2 B}$, small $p_{1 B}$ ). Thus, below, norm 1 is always the S-typical cultural norm and norm 2 is always the B-typical cultural norm. The two groups represent culturally-distinct ethnic groups. In this model, each norm is always initially present in both groups, and the question of norm origin is left for future study. 
2.2. Intra- and inter-ethnic interaction. Each time step $t$ is divided into an interaction phase and a copying phase. During the interaction phase, a fraction $m_{S}$ of group $\mathrm{S}$ crosses the group boundary and may interact with members of group $\mathrm{B}$ (inter-group interaction). We call these individuals group $\mathrm{S}$ visitors. A fraction $1-m_{S}$ remains in group $\mathrm{S}$, and we call these individuals group $\mathrm{S}$ residents. Similarly, a fraction $m_{B}$ of group B crosses the boundary into group $\mathrm{S}$ (group B visitors), while a fraction $1-m_{B}$ remains in group B (group B residents). Here we assume that individuals composing the fraction of visitors from each group $\left(m_{S}\right.$ and $\left.m_{B}\right)$ are chosen at random. In Appendix Section F we show the consequences of non-randomly selecting the composition of visitors. In addition to inter-group interactions, all individuals can also engage in intra-group interactions, e.g., group $\mathrm{S}$ visitors may interact with other group $\mathrm{S}$ visitors after arriving in group B. We modify this assumption in Appendix Section E.

Inter- and intra-group interactions are modelled as coordination games among pairs of individuals, with payoff matrices given in Table 1. If two individuals with different norms interact, they both receive a payoff of 0 . If interacting individuals from the same group have the same norm, they both receive a payoff of 1, i.e., they coordinate. However, inter-group coordination (using either norm) entails an additional marginal benefit $g_{k}$ to an individual from group $k$ (where $k=\mathrm{S}$ or $\mathrm{B}$ ). When $g_{k}>0$, inter-group coordination is more profitable than intra-group coordination for an individual from group $k$. The marginal benefits to inter-group coordination may be asymmetrical across groups. Thus, if $g_{S}$ and $g_{B}$ are the marginal inter-group coordination payoffs to individuals from groups $\mathrm{S}$ and $\mathrm{B}$, respectively, it may be the case that $g_{S} \neq g_{B}$. In Appendix Section H, we show that such asymmetries can represent differences in resource control and bargaining power between the two groups. In particular, the larger $g_{S}$ is relative to $g_{B}$, the lower the bargaining power of $\mathrm{S}$ individuals relative to $\mathrm{B}$ individuals. There is no theoretical constraint on the values of $g_{S}$ and $g_{B}$, and the model allows investigation of any range and combination of values for these parameters.

TABLE 1. Payoffs in intra- and inter-group coordination interactions for a member of group $\mathrm{S}$

\begin{tabular}{ccccc} 
& \multicolumn{4}{c}{ Person 2 norm } \\
\cline { 2 - 5 } Person 1 norm & Norm 1 & Norm 2 & Norm 1 & Norm 2 \\
\hline Norm 1 & 1 & 0 & $1+g_{S}$ & 0 \\
Norm 2 & 0 & 1 & 0 & $1+g_{S}$ \\
\hline \multicolumn{4}{c}{ Intra-ethnic interaction } & Inter-ethnic interaction ${ }^{\mathrm{a}}$ \\
a Person 1 from group S interacts with Person 2 from group B. Payoffs are \\
shown for Person 1. Payoffs to Person 2 from this interaction are identical \\
after substituting $g_{B}$ for $g_{S}$.
\end{tabular}

When deciding who to interact with, we assume that individuals have an ability $0 \leq a \leq 1$ to choose interaction partners who have a norm matching theirs. McElreath et al. (2003) showed that arbitrary markers can evolve as a solution to this problem 
of assorting on norms that are difficult to directly observe. In Appendix Section G, we explicitly incorporate markers into our model and show that the qualitative conclusions below are unchanged.

We note three strong simplifying assumptions regarding assortment and coordination interactions. First, this model assumes that assortment on norm is unaffected by the frequency of norms in the population. In other words, when $a$ is large, two individuals with a rare norm can find each other to coordinate just as easily as two individuals with a common norm. We modify this assumption in Appendix Section G. Second, we assume that the populations of both groups are large enough that the process of choosing an interaction partner is reasonably approximated by a process of sampling with replacement. Third, in this model, we assume that each individual engages in exactly one interaction during the interaction phase of each time step.

To construct a payoff expression, we first distinguish among associations during the interaction phase of the model:

$$
\begin{aligned}
R_{S} & =\left(1-m_{S}\right)+d_{B} m_{B} \\
V_{S} & =m_{S}+d_{B}\left(1-m_{B}\right)
\end{aligned}
$$

These associations are shown graphically in Fig 1, and represent changes in group composition during the interaction phase. From the perspective of an individual from group $\mathrm{S}, R_{S}$ is the size of the interaction association comprising all group $\mathrm{S}$ residents and all group B visitors, expressed as a multiple of the size of group S. $V_{S}$ is the size of the interaction association comprising all group $\mathrm{S}$ visitors and all group $\mathrm{B}$ residents, expressed as a multiple of the size of group S. From the perspective of an individual from group B, all group subscripts in equations 1 and 2 are reversed.

We also define subsets of $R_{S}$ and $V_{S}$ :

$$
\begin{aligned}
R_{1 S} & =p_{1 S}\left(1-m_{S}\right)+p_{1 B} d_{B} m_{B} \\
V_{1 S} & =p_{1 S} m_{S}+p_{1 B} d_{B}\left(1-m_{B}\right)
\end{aligned}
$$

$R_{1 S}$ is the number of individuals in $R_{S}$ who have norm 1 , expressed as a multiple of the size of group S. $V_{1 S}$ is the number of individuals in $V_{S}$ who have norm 1, expressed as a multiple of the size of group S. From the perspective of an individual from group B, the indices representing groups in equations 3 and 4 are reversed. From the perspective of an individual with norm 2, the indices representing norms are reversed.

Given these interaction associations, the payoff to a focal individual with norm 1 from group $\mathrm{S}$ is 


$$
\begin{gathered}
w_{1 S}=a\left\{\left(1-m_{S}\right)\left[\frac{p_{1 S}\left(1-m_{S}\right)}{R_{1 S}}(1)+\frac{p_{1 B} d_{B} m_{B}}{R_{1 S}}\left(1+g_{S}\right)\right]+\right. \\
\left.m_{S}\left[\frac{p_{1 S} m_{S}}{V_{1 S}}(1)+\frac{p_{1 B} d_{B}\left(1-m_{B}\right)}{V_{1 S}}\left(1+g_{S}\right)\right]\right\}+ \\
(1-a)\left\{\left(1-m_{S}\right)\left[\frac{p_{1 S}\left(1-m_{S}\right)}{R_{S}}(1)+\frac{p_{1 B} d_{B} m_{B}}{R_{S}}\left(1+g_{S}\right)\right]+\right. \\
\left.m_{S}\left[\frac{p_{1 S} m_{S}}{V_{S}}(1)+\frac{p_{1 B} d_{B}\left(1-m_{B}\right)}{V_{S}}\left(1+g_{S}\right)\right]\right\}
\end{gathered}
$$

This payoff expression is explained in Appendix Section A. Payoffs to individuals with norm 2 are obtained by reversing all norm indices. Payoffs to individuals in group B are obtained by reversing all group indices.

2.3. Copying of norms. At the conclusion of the interaction phase, all inter-group visitors (composing the fractions $m_{S}$ and $m_{B}$ ) return to their respective home groups. Individuals then have the opportunity to copy the norm of another individual in their group. We assume the replicator dynamic for copying (McElreath and Boyd 2007, pg 29-31), such that individuals choose another individual in their group at random and bias their probability of copying that person's norm phenotype on the average payoff difference between their phenotype and the other person's phenotype during the previous interaction step. A parameter $\mu$ scales the strength of the copying bias with the difference in average payoffs between norms. Note that if payoffs to individuals are public information (e.g., wealth or favor in a small-scale society where gossip is common: Gluckman 1963, Wiessner 2005), prestige- or success-biased copying may occur (Henrich and Gil-White 2001), such that the choice of potential copying partners is non-random. We do not model such a mechanism here, as we wish to isolate the effect of inter-group payoff asymmetries on norm dynamics, which is our focus. Incorporating these copying assumptions and the payoff expression above into a recursion for changing norm frequencies in group $\mathrm{S}$ yields

$$
p_{1 S}^{\prime}=p_{1 S}+p_{1 S} p_{2 S} \mu\left(w_{1 S}-w_{2 S}\right)
$$

where $p_{1 S}^{\prime}$ is the frequency of norm 1 in group $\mathrm{S}$ in the next time step. This recursion is derived in Appendix Section B.

\section{Results}

\subsection{General analysis.}

3.1.1. Perfect assortment on norm. Substituting equation 5 into equation 6 and subtracting $p_{1 S}$ yields the difference equation for the frequency of norm 1 in group $\mathrm{S}$. If individuals assort perfectly on norm $(a=1)$, we show in Appendix Section $\mathrm{C}$ that this simplifies to

$$
\Delta p_{1 S}=-\mu g_{S} d_{B} p_{1 S} p_{2 S}\left\{\left(1-m_{S}\right)\left[\left(1-m_{S}\right) m_{B} \beta_{R_{S}}\right]+m_{S}\left[m_{S}\left(1-m_{B}\right) \beta_{V_{S}}\right]\right\}
$$

where $\beta_{R_{S}}$ and $\beta_{V_{S}}$ are coefficients of the regressions of group on norm in the interaction associations $R_{S}$ and $V_{S}$, respectively. Note that each $\beta$ term is a function of the norm frequencies in both groups, and thus the $\beta$ s are not constants. 
Equation 7 says that, when assortment on norm is perfect, the rate of change of the frequency of norm 1 in group $\mathrm{S}\left(\left|\Delta p_{1 S}\right|\right)$ increases with stronger payoff-bias in copying norms from in-group members $(\mu)$, a larger advantage to group $\mathrm{S}$ members of intergroup over intra-group coordination $\left(g_{S}\right)$, a larger relative size of group $\mathrm{B}\left(d_{B}\right)$, and greater variance in norms within group $\mathrm{S}\left(p_{1 S} p_{2 S}\right)$. A proportion $1-m_{S}$ of $\Delta p_{1 S}$ is affected by payoffs in the interaction association $R_{S}$, and a proportion $m_{S}$ is affected by payoffs in $V_{S}$. The sign of $\Delta p_{1 S}$ is determined by the signs of the relative payoffs in each interaction association (see Appendix Section C). For instance, in group S, norm 1 increases when rare if it is common in group $\mathrm{B}$ (both $\beta$ s are negative, making $\Delta p_{1 S}$ positive). Conversely, in group $\mathrm{S}$, norm 1 decreases when common if it is rare in group B (both $\beta$ s are positive, making $\Delta p_{1 S}$ negative). Thus, two corner equilibria, where one norm reaches fixation in one group while the other norm reaches fixation in the other group, are unstable (demonstrated by numerical analysis in Appendix Fig $4 \mathrm{D})$. As the frequency of norm 1 in group $\mathrm{S}$ approaches the frequency of norm 1 in group $\mathrm{B}, \Delta p_{1 S}$ approaches 0 (both $\beta$ s approach 0 ). Thus, when assortment on norm is perfect, equation 7 reveals stable mixed equilibria in which both norms are present in each group (shown in Appendix Fig 4D). The equilibria are determined by the ratios $g_{S} / g_{B}$ and $d_{B} / d_{S}$ when comparing equation 7 with an analogous equation for $\Delta p_{1 B}$ (i.e., reverse group subscripts in equation 7). For instance, if both ratios are $>1$, and norm 1 is common in $\mathrm{S}$ and rare in $\mathrm{B}$, then the equilibrium frequency of norm 1 in both groups is $<0.5$, as $\left|\Delta p_{1 S}\right|>\left|\Delta p_{1 B}\right|$. In other words, $p_{1 S}$ decreases faster than $p_{1 B}$ increases. The two frequencies will coincide at a value $<0.5$, at which point $\Delta p_{1 S}=\Delta p_{1 B}=0$ and equilibrium is reached.

3.1.2. Random interaction with respect to norm. If individuals interact at random with respect to norm $(a=0)$, then substituting equation 5 into equation 6 and subtracting $p_{1 S}$ yields

$$
\begin{gathered}
\Delta p_{1 S}=\mu p_{1 S} p_{2 S}\left\{\left(1-m_{S}\right)\left[\frac{\left(1-m_{S}\right)\left(p_{1 S}-p_{2 S}\right)+d_{B} m_{B}\left(1+g_{S}\right)\left(p_{1 B}-p_{2 B}\right)}{R_{S}}\right]+\right. \\
\left.m_{S}\left[\frac{m_{S}\left(p_{1 S}-p_{2 S}\right)+d_{B}\left(1-m_{B}\right)\left(1+g_{S}\right)\left(p_{1 B}-p_{2 B}\right)}{V_{S}}\right]\right\}
\end{gathered}
$$

This equation is interpretable without reducing to the form in equation 7 . The first inner term in brackets in equation 8 is the difference in expected payoffs between an individual from group $\mathrm{S}$ who has norm 1 and an individual from group $\mathrm{S}$ who has norm 2, within the interaction association $R_{S}$. Similarly, the second term in brackets is the difference in expected payoffs between an individual from group $\mathrm{S}$ who has norm 1 and an individual from group $\mathrm{S}$ who has norm 2 , within the interaction association $V_{S}$. Both of these terms are positive when norm 1 is common in both groups $\left(p_{1 S}\right.$ and $p_{1 B}>0.5$ ). They become larger as norm 1 increases in frequency, especially if $g_{S}$ and $d_{B}$ are also large. If a norm is common in one group but rare in the other, the dynamics are most sensitive to the frequency of the norm in the larger group (e.g., group $\mathrm{B}$ if $d_{B}$ is large), especially if successfully coordinating with members of that group yields a high payoff (e.g., $g_{S}$ is large). The only stable equilibria occur at the corners, where one norm (not necessarily the same one) goes to fixation in each group (shown in Appendix in Fig 4A). 
Similar to equation 7 above, $\Delta p_{1 S}$ is larger in magnitude when payoff-biased copying $(\mu)$ and within-group variance in norms $\left(p_{1 S} p_{2 S}\right)$ are large. Also similar to equation 7 , the rates of change of norm frequencies approaching equilibrium are dependent on the frequencies of visitors from each group. For instance, if there are no visitors from the majority group $\mathrm{B}\left(m_{B}=0\right)$, the most common norm in group $\mathrm{S}$ increases in frequency among $\mathrm{S}$ residents. However, $\mathrm{S}$ visitors are maximally sensitive to the most common norm in group B, which may be different. As long as there are few $\mathrm{S}$ visitors $\left(m_{S}\right.$ is small), norm dynamics in group $\mathrm{S}$ will be most heavily influenced by norm frequencies among $\mathrm{S}$ residents. If there are no $\mathrm{S}$ visitors $\left(m_{S}=0\right)$, then the most common norm in group $\mathrm{S}$ increases in frequency unless group $\mathrm{B}$ sends many visitors $\left(d_{B} m_{B}\right.$ is large) and/or $\mathrm{S}$ individuals receive a large payoff from successfully coordinating with members of group B ( $g_{S}$ is large). In that case, the most common norm in group B may increase in frequency within group $\mathrm{S}$. If there is no inter-group interaction $\left(m_{S}=m_{B}=0\right)$, then the most common norm in each group goes to fixation within that group.

When assortment on norms is neither perfect nor completely random $(0<a<1)$, equilibrium frequencies of norms in the two groups will be a mixture of the two types of dynamics described above. Below we use a numerical analysis to examine the frequencies of norms under conditions most relevant for the cultural contexts of interest.

3.2. Numerical analysis. Mathematica notebooks containing numerical analyses, equilibrium solutions, and figures in this paper are available on Github at github.com/jabunce/buncemcelreath-2017-norm-model.

3.2.1. Bi-directional visiting. When visitors from each group travel to interact with the other $\left(m_{S}=m_{B} \neq 0\right)$, and assortment on norm is high (large $\left.a\right)$, the norm typical of group $\mathrm{S}$ goes to fixation in both groups if $\mathrm{S}$ individuals receive much lower marginal coordination payoffs than B individuals $\left(g_{S}<<g_{B}\right)$ (upper left quadrants of plots in Fig $2 \mathrm{~A}$ and $\mathrm{B}$ ). Otherwise this norm is lost from the population (upper regions in Fig $2 \mathrm{~A}$ and $\mathrm{B}$ ). If interaction is more random with respect to norm (low $a$ ), the initially most common norm in each group (if there are few visitors, Fig 2A) or the initially most common norm in the larger group (if there are many visitors, Fig 2B) goes to fixation in each group (example in Appendix Fig 4A). Because of the benefits to coordination, in the real world we expect people to be very interested in detecting the norms of potential interaction partners and assorting non-randomly, and we expect selection to favour such an interest and ability (McElreath et al. 2003). Thus, in this analysis, we focus on conditions of moderate to large $a$. Because of the benefits to inter-group coordination over intra-group coordination, we may also expect people to preferentially interact with individuals who have their norm but are not from their group. In Appendix Section E we show that such a preference has little effect on the results when visiting is relatively low, and makes it somewhat easier to maintain the S-typical norm 1 when visiting is high (Appendix Fig 5A and B).

In this model, when assortment on norm is high, individuals who have a norm common in the other group have a high probability of successful, and profitable, inter-group coordination. At the same time, S individuals, due to their smaller numbers, have a higher probability of attempting inter-group (as opposed to intra-group) 
coordination than do B individuals. For this reason, S individuals with B-typical norm 2 increase in group $\mathrm{S}$ faster than B individuals with S-typical norm 1 increase in group B, all else equal. The end result is fixation of the B-typical norm in the population. To overcome this effect, B individuals must receive a substantially higher inter-group coordination payoff than S individuals. In that case, the S-typical norm can go to fixation in both groups. Narrative explanations of model dynamics here and below, as well as analysis of frequency trajectories and equilibrium solutions, are provided in Appendix Section D.

3.2.2. One-directional visiting. When $\mathrm{S}$ individuals can travel to group $\mathrm{B}$ to interact but B individuals cannot travel to group $\mathrm{S}\left(m_{S}>0\right.$ and $\left.m_{B}=0\right)$, and assortment on norm is high, the S-typical norm 1 can go to fixation in both groups even if $\mathrm{S}$ individuals receive higher marginal coordination payoffs than B individuals $\left(g_{S}>g_{B}\right)$ (upper regions in Fig $2 \mathrm{C}$ and $\mathrm{D}$ ). As assortment on norm is lowered, the initially most common norm in each group attains high frequency if there are relatively few visitors from group S (lower regions in Fig 2C), though S-typical norm 1 can persist in group B at low frequency (shown more clearly in Appendix Figs $3 \mathrm{C}$ and $4 \mathrm{~F}$ ). When assortment on norm is low and there are many $\mathrm{S}$ visitors, S-typical norm 1 can be preserved only when S individuals receive lower marginal coordination payoffs (lower regions in Fig 2D). In Appendix Section E we show that these results hold when individuals preferentially attempt coordination with individuals who are not from their group (Appendix Fig 5C and D).

In this model, eliminating visitors from one of the two groups increases the frequency of intra-group interactions relative to inter-group interactions. When assortment on norm is high but imperfect, $\mathrm{S}$ residents with B-typical norm 2 receive relatively low average payoffs within their own group, where their norm is uncommon and there are no B visitors with whom to engage in inter-group coordination. The few $\mathrm{S}$ visitors with norm 2 achieve higher inter-group coordination payoffs but can do little to offset this effect, as their payoffs are diluted into the average payoff for norm 2 upon their return home to group S (though see caption for Fig 2C). Meanwhile, $\mathrm{B}$ individuals with S-typical norm 1 receive high average payoffs as a result of intergroup coordination. There is no payoff dilution effect and they increase quickly in frequency. Consequently S-typical norm 1 increases in group B faster than B-typical norm 2 increases in group S, and norm 1 can go to fixation in both groups. See Appendix Section D for further explanation and analysis.

\section{Discussion}

We have examined the dynamics of cultural norms in a group-structured population where inter-group coordination is more profitable than intra-group coordination. Modifying the degree of assortment on norm, along with asymmetries in the marginal payoffs to inter-group coordination, is sufficient to generate a range of outcomes, including the maintenance of group-level norm variation or the replacement of one group's norms by those of another. Previous evolutionary models of cultural change in group-structured populations show that the evolution of group-level cultural variation can make inter-group interaction costly, and thus infrequent (e.g., McElreath et al. 2003). Such variation may be maintained if groups are balanced in population growth rate (e.g., Boyd and Richerson 1990) and prestige (e.g., Boyd and Richerson 
2002). In contrast, in our model inter-ethnic coordination is both profitable and frequent, groups are asymmetric in size (though demographically static), and individuals from different groups differ in bargaining power (a potential correlate of prestige). We believe that these conditions better approximate many real-world contexts of ethnic interaction. Under such conditions, we show that group-level variation in cultural norms can often be maintained if inter-ethnic interaction is moderate, and individuals do not assort strongly on norm (i.e., low $a$ in Fig 2A and C). However, such a situation is rarely desirable, as nearly all inter-ethnic interaction then results in norm miscoordination, negating potentially profitable exchange of resources or services between groups.

In a more likely scenario, the norms of one group replace those of another in the domain of interaction. In other words, individuals from the two groups adopt a single norm for interaction in a given domain, facilitating mutually-profitable inter-ethnic (as well as intra-ethnic) coordination. In our model, this often occurs if people can assort strongly, but imperfectly, on norm (or a marker that covaries with norm), an ability that is usually adaptive (McElreath et al. 2003). The question then becomes, which group-typical norm will replace the other? Our model shows that the norm of the larger group will usually go to fixation in both groups unless minority-group individuals receive much lower inter-ethnic coordination benefits (high $a$ and low $g_{S}$ in Fig 2A), i.e., they have much higher bargaining power (see Appendix Section H).

However, an important prediction of this model is that the range of conditions favouring the fixation in both groups - and thus, the sustainability - of minoritytypical cultural norms is also increased if members of the minority group engage in substantial inter-ethnic interaction, yet live in a place that majority individuals cannot or do not want to visit. Under these conditions, maintenance of such minority cultural traits can occur even if minority individuals benefit more from inter-group coordination than do majority individuals. In Appendix Section H we show that such an asymmetry in coordination benefits corresponds to a situation in which minority individuals control fewer resources and have low bargaining power. Thus, we predict that, in the absence of other processes affecting cultural change, the sustainability of certain cultural traits of a dis-empowered ethnic minority may be enhanced if they have a homeland that members of a powerful majority do not wish to visit, or with a border permeable to minority but not majority members.

Cultural change in any real-world context is surely the result of a complex amalgam of many mechanisms interacting across scales of organization (e.g., individual psychology to state-level institutions). Our simple model purposefully excludes nearly all of this complexity in order to examine the logical consequences of one such mechanism in isolation. Consequently, the model can only approximate a particular ethnographic context in a very crude sense. However, the exercise of viewing the world through the lens of the model can draw our attention to measurable attributes of a population and its inter-individual interactions that may play some causal role in generating observed patterns of cultural change, and thus focus further empirical exploration. We attempt such an exercise here. Based on fieldwork by one of us (JAB) in an Amazonian population, we have previously suggested that inter-ethnic differences in coordination benefits (bargaining power) may explain, at least in part, current distributions of minority indigenous cultural norms in the domains of education, wage 
labour, and commerce (Bunce and McElreath 2017). Below we show how the above model can contribute to our understanding of the consequences of such asymmetrical benefits for long-term cultural sustainability or change in this population, given observed forms of inter-ethnic interaction.

4.1. Applying the model in an Amazonian context. In the Department of Madre de Dios, in Amazonian Peru, indigenous Matsigenka have cultural norms that differ from those of majority Mestizo colonists in many domains, including education (see Bunce and McElreath 2017). Matsigenka students travel to enrol tuition-free in boarding secondary schools run by Mestizos in distant towns ( $10 \%$ of adults in one Matsigenka community have attended, thus $m_{\text {Matsi }} \approx 0.1$ ). In contrast, Mestizos usually cannot enter Matsigenka communities located inside a protected national park $\left(m_{\text {Mest }}=0\right)$. Payoffs to inter-ethnic educational coordination consist of students receiving desired knowledge, while teachers benefit from student enrolment, upon which a teacher's employment in the regional educational system heavily depends. In these schools, Matsigenka children make up a minority of the student body. Most value their Western education highly and are happy to be there, suggesting that they place less value on the non-Western education they would receive from co-ethnics in their community had they not enrolled in the boarding school (i.e., $g_{\text {student }}>0$ ). In each individual student-teacher coordination, the knowledge received by the student is likely more valuable to her than is the enrolment of a single student to a (hypothetically dispassionate) teacher with a large class whose benefits depend primarily on maintaining a class size sufficient to justify employment. Thus, $g_{\text {student }}>g_{\text {teacher }}$, and teachers have power over students because they wield a credible threat of expulsion (see Appendix Section H). We make four additional assumptions: 1) Western secondary-school education is valued by all Matsigenka; 2) Matsigenka boarding school students who have Mestizo-typical pedagogical norms learn the most in the boarding school, thereby obtaining the greatest relative education-related benefits of anyone in the population, on average (analogous to $\mathrm{S}$ visitors when $g_{S}>g_{B}$ ); 3 ) individuals tend to copy the pedagogical norms of successful co-ethnics $(\mu>0)$; and 4) boarding school students return to their home communities. Note that all of these assumptions are amenable to empirical testing.

Given this ethnographic context, plausible long-term cultural dynamics could entail either the maintenance of Matsigenka-typical pedagogical norms within the Matsigenka communities, or their replacement by Mestizo-typical norms. To the extent that this context is approximated by our model for one-directional visiting (e.g., Fig $2 \mathrm{C}$ and $\mathrm{D}$ ), we would predict that Matsigenka-typical pedagogical norms are maintained at high frequency, as long as people prefer to engage in teaching-learning interactions with others who have norms like their own (i.e., large $a$ ). If such cultural sustainability is in fact observed, we can look for empirical evidence of a mechanism suggested by the one-directional visiting model: Matsigenka with Mestizo-typical pedagogical norms are rare and so do poorly when they are in, or return to, the Matsigenka communities. It is thus in the perceived self-interest of all Matsigenka (and perhaps some Mestizo teachers) to retain, adopt, or re-learn Matsigenka-typical norms of education. However, if it is observed that Matsigenka-typical pedagogical norms are being lost from the Matsigenka communities despite the reasonableness of the above assumptions, a mechanism other than that modeled here may be exerting 
a more powerful influence on the cultural dynamics of the population (e.g., one of the alternative mechanisms examined by Bunce and McElreath 2017).

4.2. Conclusions. As shown, the model we develop here can be used to generate empirical predictions that serve as baseline hypotheses for researchers interested in cultural change in ethnically-structured populations. When predictions are not supported, we can direct our attention to violations of the core assumptions of the model, and to other mechanisms that may be responsible for the observed cultural dynamics. If the principle driver of cultural dynamics in this model, i.e., ethnic asymmetries in benefits to inter-ethnic coordination, is shown to be an important component of cultural change across a range of ethnographic contexts, it would suggest one plausible strategy for the sustainability of cultural norms valued by an ethnic minority that wishes to continue active engagement with a powerful majority group: establish a territory with a border that minority individuals can cross freely, but that restricts the inflow of majority individuals. We encourage other researchers interested in cultural change to modify the parameter values, and perhaps the structure, of our model simulations in order to generate predictions appropriate for their particular ethnographic context. It is our belief that such a dialectic between formal theory and empirical work is necessary to advance understanding of the complex processes of cultural change in our species.

\section{Code and Data Availability}

Wolfram Mathematica (version 10.2.0.0) notebooks containing numerical analyses, equilibrium solutions, and figures in this paper are available on Github at github.com/jabunce/buncemcelreath-2017-norm-model.

\section{ACKNOWLEDGMENTS}

Anne Kandler helped develop the model in Appendix Section E. A question by Monique Borgerhoff-Mulder inspired the model in Appendix Section F. Bret Beheim helped with the derivation of equation 7. Anne Pisor, Anne Kandler, Cristina Moya, Ben Purzycki, Cody Ross, Caissa Revilla, and staff in the Department of Human Behavior, Ecology, and Culture at the Max Planck Institute for Evolutionary Anthropology provided valuable comments and criticism on early versions of this paper. Funding was provided by the U.S. National Science Foundation (BCS 1227152) to JAB, and by the Max Planck Society. Any error in word, math, or script is ours.

\section{AUTHOR CONTRIBUTIONS}

$\mathrm{JAB}$ and RM designed the research. JAB performed the analysis. JAB and RM wrote the paper.

Corresponding author: John Andrew Bunce, john_bunce@eva.mpg.de 
Group S

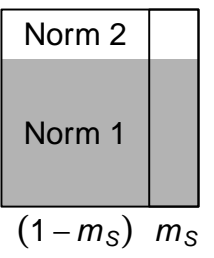

Interaction Association $R_{S}$

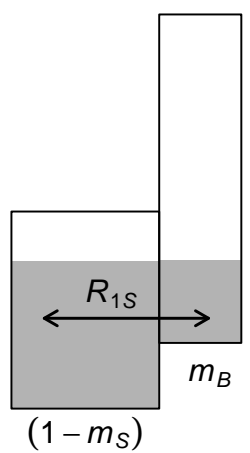

Group B

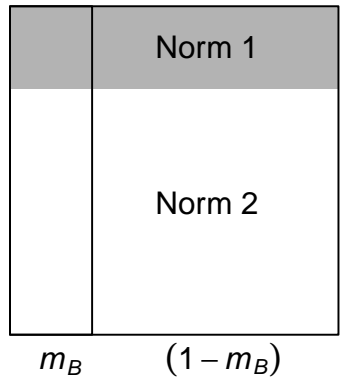

Interaction Association $V_{S}$

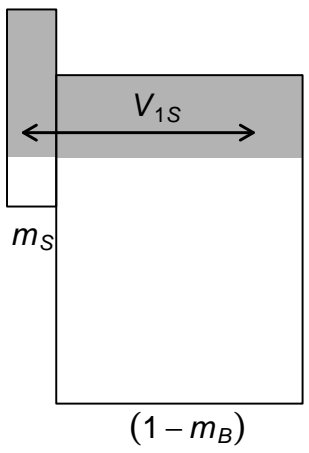

FiguRE 1. Schematic representation of a group-structured population where group $\mathrm{S}$ (minority) and group B (majority) differ in the frequency of norm 1 (and its alternative, norm 2). A proportion $m_{S}$ of group $\mathrm{S}$ consists of individuals who will leave to visit group B during the subsequent interaction phase (lower half of the figure). We call these group $\mathrm{S}$ individuals visitors, and the rest residents. Group B consists of its own visitors and residents. During the interaction phase, four associations are formed. $R_{S}$ is the association of all group $\mathrm{S}$ residents and group $\mathrm{B}$ visitors. $V_{S}$ is the association of all group $\mathrm{S}$ visitors and group B residents. $R_{1 S}$ and $V_{1 S}$ are, respectively, associations of only those members of $R_{S}$ and $V_{S}$ that have norm 1 (see equations 1-4). Note that, from the perspective of group $\mathrm{B}$, the $V$ and $R$ designations for the interaction associations are exchanged. After the interaction phase, all visitors return to their original groups for the copying phase. 

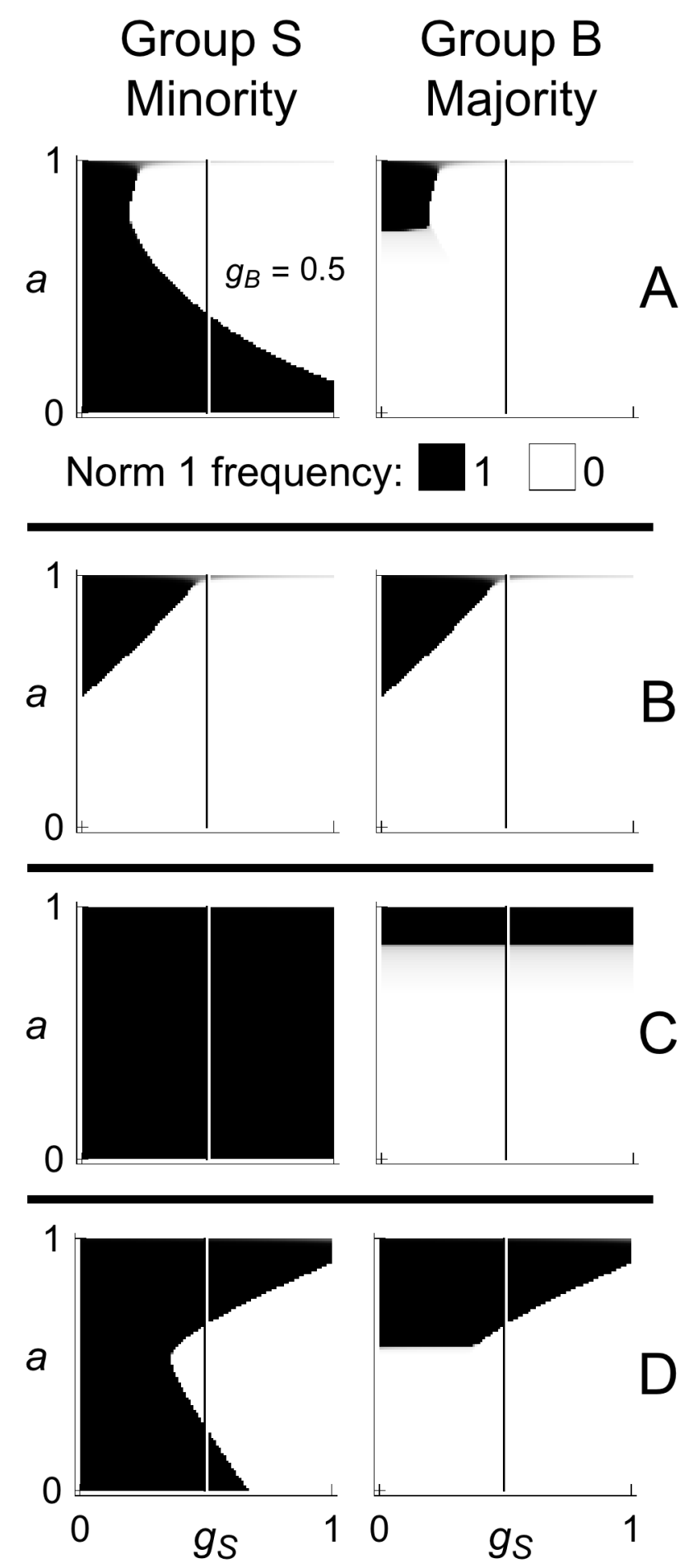

Figure 2. (Caption next page.) 
Figure 2. (Previous page) Long-run frequency of minority-typical norm 1 in minority ( $\mathrm{S}$, first column) and majority (B, second column) groups after 400 simulated time steps, given a range of assortment probabilities $(a)$ and inter-group coordination benefits to $\mathrm{S}$ individuals $\left(g_{S}\right)$. For all simulations, $d_{S}=0.5, \mu=0.5, g_{B}=0.5$ (indicated by vertical lines in plots), and norm 1 initially occurs at high frequency (0.9) in group $\mathrm{S}$ and low frequency (0.1) in group B. Black areas in plots correspond to fixation of S-typical norm 1, while white areas correspond to loss of norm 1. A) Low bi-directional inter-group visiting $\left(m_{S}=m_{B}=0.1\right)$. If assortment is high (large $\left.a\right)$, S-typical norm 1 goes to fixation in both groups if $\mathrm{B}$ individuals benefit most from inter-group coordination $\left(g_{B}=0.5>>g_{S}\right)$, and is lost from both groups if the $\mathrm{S}$ individuals benefit most $\left(g_{S}>g_{B}=0.5\right)$. B) High bi-directional inter-group visiting $\left(m_{S}=m_{B}=0.4\right)$. If assortment $(a)$ is high and $\mathrm{B}$ individuals benefit most from inter-group coordination $\left(g_{B}=0.5>g_{S}\right)$, the conditions under which norm 1 goes to fixation in both groups broaden slightly. C) Visitors are restricted from group B but not group $\mathrm{S}\left(m_{B}=0\right.$ and $\left.m_{S}=0.1\right)$. S-typical norm 1 is always maintained in group $\mathrm{S}$, and can go to fixation in group $\mathrm{B}$ as well if assortment is high enough (e.g., $>0.8$ ). This occurs regardless of the value of $g_{S}$ over the range 0 to 1 . However, note that the $g$ 's have no theoretically-constrained range, and, under these conditions, very large values (e.g., $g_{S}>3$ ) would in fact change the long-run dynamics. D) Same conditions as C, but now $\mathrm{S}$ visitors to group B are increased $\left(m_{S}=0.4\right)$. The scope for maintenance of norm 1 in group $\mathrm{S}$ is decreased, especially when $\mathrm{S}$ individuals benefit most from inter-group coordination $\left(g_{S}>g_{B}=0.5\right)$. Note that, although not apparent, at $a=1$ in all plots both norm 1 and norm 2 are maintained in both groups at equal (e.g., equally high or low) frequencies at equilibrium. These mixed equilibria can be seen more clearly in Fig 4D. 


\section{REFERENCES}

Aikman, S. (2003). La Educación Indígena en Sudamérica: Interculturalidad y Bilingüismo en Madre de Dios, Perú. IEP Ediciones, Lima.

Baer, G. (2004). ¿Comes carne de armadillo? acerca del problema de la identidad de los Matsiguenka del Bajo Urubamba en el Oriente Peruano. Boletín de Antropología (Universidad de Antioquia), 18(35):317-336.

Barth, F. (1998). Introduction. In Barth, F., editor, Ethnic Groups and Boundaries: The Social Organization of Culture Difference, pages 9-38. Waveland Press, Inc., Long Grove.

Bicchieri, C. (2006). The Grammar of Society: The Nature and Dynamics of Social Norms. Cambridge University Press, Cambridge.

Bowles, S. and Gintis, H. (1993). The revenge of Homo economicus: contested exchange and the revival of political economy. Journal of Economic Perspectives, $7(1): 83-102$.

Boyd, R. and Richerson, P. J. (1987). The evolution of ethnic markers. Cultural Anthropology, 2(1):65-79.

Boyd, R. and Richerson, P. J. (1990). Group selection among alternative evolutionarily stable strategies. Journal of Theoretical Biology, 145(331-342).

Boyd, R. and Richerson, P. J. (2002). Group beneficial norms can spread rapidly in a structured population. Journal of Theoretical Biology, 215:287-296.

Boyd, R. and Richerson, P. J. (2009). Voting with your feet: payoff biased migration and the evolution of group beneficial behavior. Journal of Theoretical Biology, 257:331-339.

Bunce, J. A. and McElreath, R. (2017). Interethnic interaction, strategic bargaining power, and the dynamics of cultural norms: a field study in an Amazonian population. Human Nature, 28(4):434-456.

Ensminger, J. and Knight, J. (1997). Changing social norms: common property, bridewealth, and clan exogamy. Current Anthropology, 38(1):1-24.

Gilpin, W., Feldman, M. W., and Aoki, K. (2016). An ecocultural model predicts neanderthal extinction through competition with modern humans. Proceedings of the National Academy of Sciences of the USA, 113(8):2134-2139.

Gluckman, M. (1963). Papers in honor of Melville J. Herskovits: Gossip and scandal. Current Anthropology, 4(3):307-316.

Henrich, J. and Gil-White, F. J. (2001). The evolution of prestige: freely conferred deference as a mechanism for enhancing the benefits of cultural transmission. Evolution and Human Behavior, 22:165-196.

Knight, J. (1992). Institutions and Social Conflict. Cambridge University Press, Cambridge.

Kopenawa, D. and Albert, B. (2013). The Falling Sky: Words of a Yanomami Shaman. The Belknap Press of Harvard University Press, Cambridge.

McElreath, R. and Boyd, R. (2007). Mathematical Models of Social Evolution: A Guide for the Perplexed. University of Chicago Press, Chicago.

McElreath, R., Boyd, R., and Richerson, P. J. (2003). Shared norms and the evolution of ethnic markers. Current Anthropology, 44(1):122-129.

Nagel, J. (1996). American Indian Ethnic Renewal: Red Power and the Resurgence of Identity and Culture. Oxford University Press, New York. 
Nave, A. (2000). Marriage and the maintenance of ethnic group boundaries: the case of Mauritius. Ethnic and Racial Studies, 23(2):329-352.

Pisor, A. C. and Gurven, M. (2016). Risk buffering and resource access shape valuation of out-group strangers. Scientific Reports, 6(30435).

Richerson, P. J. and Boyd, R. (2005). Not by Genes Alone: How Culture Transformed Human Evolution. University of Chicago Press, Chicago.

Ross, R. M. and Atkinson, Q. D. (2016). Folktale transmission in the Arctic provides evidence for high bandwidth social learning among hunter-gatherer groups. Evolution and Human Behavior, 37:47-53.

Sahlins, M. (1994). Cosmologies of capitalism: The trans-Pacific sector of "The World System". In Dirks, N. B., Eley, G., and Ortner, S. B., editors, Culture/Power/History: A Reader in Contemporary Social Theory, book section 13, pages 412-455. Princeton University Press, Princeton.

Solway, J. S. and Lee, R. B. (1990). Foragers, genuine or spurious? situating the Kalahari San in history. Current Anthropology, 31(2):109-146.

Wiessner, P. (2005). Norm enforcement among the Ju/'hoansi Bushmen. Human Nature, 16(2):115-145.

Wolf, E. R. (1982). Europe and the People Without History. University of California Press, Berkeley. 


\title{
APPENDIX: SUSTAINABILITY OF MINORITY CULTURE WHEN INTER-ETHNIC INTERACTION IS PROFITABLE
}

\author{
JOHN BUNCE*1,2,3 AND RICHARD MCELREATH ${ }^{1,2}$
}

\footnotetext{
${ }^{1}$ Max Planck Institute for Evolutionary Anthropology, Deutscher Platz 6, 04103 Leipzig, Germany. ${ }^{2}$ Department of Anthropology, University of California, Davis, One Shields Ave, Davis CA 95616. ${ }^{3}$ Department of Anthropology, Indiana University, 701 E. Kirkwood Avenue, Bloomington, IN 47405

E-mail address: john_bunce@eva.mpg.de.
} 


\section{Appendix A. Explanation of equation 5}

Assume a focal individual with norm 1 from group S. Reading from left to right, equation 5 says that this focal individual is guaranteed to interact with a partner who shares her norm 1 with probability $a$. With probability $1-m_{S}$ the focal is a group $\mathrm{S}$ resident (i.e., does not travel to interact in group B). With probability $\frac{p_{1 S}\left(1-m_{S}\right)}{R_{1 S}}$ the focal encounters a co-ethnic group S resident with whom to interact from among the pool of individuals with norm $\mathrm{S}$ in the interaction association that comprises group $\mathrm{S}$ residents and group $\mathrm{B}$ visitors $\left(R_{1 S}\right.$, see equation 3 and Fig. 1$)$. If she encounters such an individual, she receives the intra-ethnic coordination payoff of 1 (Table 1). With probability $\frac{p_{1 B} d_{B} m_{B}}{R_{1 S}}$ the focal instead encounters a group B visitor from among the pool of individuals with norm 1 in that same interaction association. In that case, she receives the inter-ethnic coordination payoff of $1+g_{S}$. With probability $m_{S}$ the focal is a group $\mathrm{S}$ visitor (i.e., travels to interact in group B). With probability $\frac{p_{1 S} m_{S}}{V_{1 S}}$ the focal encounters a co-ethnic group $\mathrm{S}$ visitor with whom to interact from among the pool of individuals with norm 1 in the interaction association that comprises group $\mathrm{S}$ visitors and group B residents (equation 4), receiving the intra-ethnic coordination payoff of 1 . With probability $\frac{p_{1 B} d_{B}\left(1-m_{B}\right)}{V_{1 S}}$, she instead encounters a group B resident and receives the inter-ethnic coordination payoff of $1+g_{S}$. The subsequent terms have an analogous structure for the probability $(1-a)$ that the focal interacts with a partner at random with respect to norm. In this case, the interaction associations comprising group $\mathrm{S}$ residents and group $\mathrm{B}$ visitors $\left(R_{S}\right)$ and group $\mathrm{S}$ visitors and group B residents $\left(V_{S}\right)$ include all such individuals regardless of norm (see equations 1 and 2 and Fig 1$)$.

\section{Appendix B. Derivation of equation 6}

To derive equation 6 , we first construct an norm-copying table (Table 2) to keep track of the different types of encounters that may occur during the copying step of the model.

TABLE 2. Norm-copying table for individuals with norm 1 or norm 2 in group $\mathrm{S}$

\begin{tabular}{ccccc} 
Self & Other & $\operatorname{Pr}($ self, other $)$ & $\operatorname{Pr}(1 \mid \text { self, other })^{\mathrm{a}}$ & $\operatorname{Pr}(2 \mid$ self, other $)$ \\
\hline 1 & 1 & $p_{1 S}^{2}$ & 1 & 0 \\
2 & 1 & $\left(1-p_{1 S}\right) p_{1 S}$ & $0.5+\mu\left(w_{1 S}-w_{2 S}\right)$ & $0.5+\mu\left(w_{2 S}-w_{1 S}\right)$ \\
1 & 2 & $p_{1 S}\left(1-p_{1 S}\right)$ & $0.5+\mu\left(w_{1 S}-w_{2 S}\right)$ & $0.5+\mu\left(w_{2 S}-w_{1 S}\right)$ \\
2 & 2 & $\left(1-p_{1 S}\right)^{2}$ & 0 & 1 \\
\hline
\end{tabular}

${ }^{\text {a }} \operatorname{Pr}($ Self=1 after copying $\mid$ current value of Self, current value of Other). Column 5 heading interpreted analogously.

Derivation of the terms $0.5+\mu\left(w_{1 S}-w_{2 S}\right)$ can be found in McElreath and Boyd (2007, pg 29). Multiplying $\operatorname{Pr}($ self, other) by $\operatorname{Pr}(1 \mid$ self, other) and summing over all 
combinations of self and other gives the frequency of norm 1 in group $S\left(p_{1 S}\right)$ after copying:

$$
p_{1 S}^{\prime}=p_{1 S}^{2}+2 p_{1 S}\left(1-p_{1 S}\right)\left[0.5+\mu\left(w_{1 S}-w_{2 S}\right)\right]
$$

Multiplying this out yields:

$$
p_{1 S}^{\prime}=p_{1 S}^{2}+p_{1 S}\left(1-p_{1 S}\right)+p_{1 S}\left(1-p_{1 S}\right) 2 \mu\left(w_{1 S}-w_{2 S}\right)
$$

The 2 can be subsumed into $\mu$. Multiplying out equation 10, simplifying, and substituting $p_{2 S}$ for $\left(1-p_{1 S}\right)$ yields equation 6 in the text.

\section{Appendix C. Derivation of EQuation 7}

Substituting equation 5 into equation 6 and subtracting $p_{1 S}$ yields the difference equation for $p_{1 S}$. If individuals assort perfectly on norm $(a=1)$, then this simplifies to

$$
\begin{array}{r}
\Delta p_{1 S}=\mu g_{S} p_{1 S} p_{2 S}\left\{\left(1-m_{S}\right)\left[\frac{\left(1-m_{S}\right) p_{2 S}}{R_{2 S}}-\frac{\left(1-m_{S}\right) p_{1 S}}{R_{1 S}}\right]\right. \\
\left.+m_{S}\left[\frac{m_{S} p_{2 S}}{V_{2 S}}-\frac{m_{S} p_{1 S}}{V_{1 S}}\right]\right\}
\end{array}
$$

41 Finding common denominators for the two difference terms in the inner brackets 42 of equation 11 yields, respectively:

$$
\left(1-m_{S}\right) \frac{p_{2 S} R_{1 S}-p_{1 S} R_{2 S}}{R_{2 S} R_{1 S}}
$$

and

$$
m_{S} \frac{p_{2 S} V_{1 S}-p_{1 S} V_{2 S}}{V_{2 S} V_{1 S}}
$$

Using equation 3 to expand the denominator of Expr 12 yields:

$$
\left[p_{2 S}\left(1-m_{S}\right)+p_{2 B} d_{B} m_{B}\right]\left[p_{1 S}\left(1-m_{S}\right)+p_{1 B} d_{B} m_{B}\right]
$$

This is simply the frequency of norm 2 in $R_{S}$ multiplied by the frequency of norm 1 in $R_{S}$, which is equivalent to $\operatorname{var}($ norm $)$ in $R_{S}$. Similarly, the denominator of Expr 13 is equivalent to $\operatorname{var}($ norm $)$ in $V_{S}$.

Multiplying out the numerator of Expr 12 yields:

$$
\left(1-m_{S}\right)\left\{p_{2 S}\left[p_{1 S}\left(1-m_{S}\right)+p_{1 B} d_{B} m_{B}\right]-p_{1 S}\left[p_{2 S}\left(1-m_{S}\right)+p_{2 B} d_{B} m_{B}\right]\right\}
$$

This simplifies to:

$$
-\left(1-m_{S}\right) d_{B} m_{B}\left(p_{1 S} p_{2 B}-p_{2 S} p_{1 B}\right)
$$


The term $p_{1 S} p_{2 B}-p_{2 S} p_{1 B}$ is the covariance of group and norm in the population. Because selection of visitors and residents from each group is random with respect to norm, this is equivalent to the covariance of group and norm in the interaction association $R_{S}$. Thus, Expr 16 becomes:

$$
-\left(1-m_{S}\right) d_{B} m_{B}\left[\operatorname{cov}\left(\text { norm, group) in } R_{S}\right]\right.
$$

Similarly, multiplying out and simplifying the numerator of Expr 13 yields:

$$
-m_{S} d_{B}\left(1-m_{B}\right)\left[\operatorname{cov}(\text { norm, group }) \text { in } V_{S}\right]
$$

Reconstituting Exprs 12 and 13 with the new numerators and denominators yields, respectively:

$$
-\left(1-m_{S}\right) d_{B} m_{B} \frac{\operatorname{cov}(\text { norm, group }) \text { in } R_{S}}{\operatorname{var}(\text { norm }) \text { in } R_{S}}
$$

and

$$
-m_{S} d_{B}\left(1-m_{B}\right) \frac{\operatorname{cov}(\text { norm, group }) \text { in } V_{S}}{\operatorname{var}(\text { norm }) \text { in } V_{S}}
$$

The signs of these two terms are determined by the indexing of group and norm. For instance, for $\Delta p_{2 S}$ and $\Delta p_{1 B}$, both terms are positive, while both are negative for $\Delta p_{2 B}$. A covariance divided by a variance is a regression coefficient. Substituting regression coefficients into Exprs 19 and 20, and then substituting these into equation 11, yields equation 7 in the text.

When there are two groups and two norm variants, as is the case here, these $\beta$ coefficients have a maximum at 1 when all individuals in group $\mathrm{S}$ have norm 1 and all individuals in group B have norm 2 , and a minimum at -1 when all individuals in group $\mathrm{S}$ have norm 2 and all in group $\mathrm{B}$ have norm 1 . The coefficents are 0 when norm 1 is equally frequent in both groups (not necessarily at a frequency of 0.5 ). When the $\beta \mathrm{s}$ are positive, most individuals with norm 1 are members of group $\mathrm{S}$, so an individual with norm 1 in group $\mathrm{S}$ has less opportunity for inter-ethnic coordination to obtain the inter-ethnic payoff bonus $g_{S}$. When the $\beta$ s are negative, there is a greater chance that a member of group $\mathrm{S}$ with norm 1 obtains the inter-ethnic payoff bonus $g_{S}$, all else being equal. The relative influence of each $\beta$ is determined by the rates of inter-ethnic visiting from each group. For instance, the relative influence of $\beta_{R_{S}}$ compared to $\beta_{V_{S}}$ increases when there are few visitors from group $\mathrm{S}$ ( $m_{S}$ is small) but many visitors from group $\mathrm{B}$ ( $m_{B}$ is large). Thus, each of the two terms in brackets in equation 7 is the relative expected payoff (expressed as a multiple of $g_{S}$ ) to an individual with norm 1 from group $\mathrm{S}$ who finds herself in the given interaction association.

\section{ApPendix D. NARRATIVE EXPLANATIONS OF MODEL DYNAMiCS}

D.1. Bi-directional visiting: loss of the minority-typical norm. To understand why S-typical norm 1 is lost from the population when assortment $(a)$ is high and $\mathrm{S}$ individuals receive a larger benefit than B individuals from inter-ethnic coordination $\left(g_{S}>g_{B}\right)$, consider interaction in early stages of the model simulation. Initial conditions are represented in the top half of Fig 1: norm 1 is common in group S and 
rare in group B. We will first take the perspective of group S. During the interaction phase (bottom half of Fig 1), S visitors and residents with norm 2 have more opportunities to engage in inter-ethnic coordination with B individuals who have norm 2 than $\mathrm{S}$ visitors and residents with norm 1 have to engage in inter-ethnic coordination with $\mathrm{B}$ individuals who have norm 1 . This can be seen in the interaction associations in Fig 1. In $R_{S}$ there are more $\mathrm{B}$ visitors with norm 2 (white in $m_{B}$ ) for every $\mathrm{S}$ resident with norm 2 (white in $1-m_{S}$ ) than there are B visitors with norm 1 (grey in $m_{B}$ ) for every $\mathrm{S}$ resident with norm 1 (grey in $1-m_{S}$ ). Similarly, in $V_{S}$ there are more $\mathrm{B}$ residents with norm 2 (white in $1-m_{B}$ ) for every $\mathrm{S}$ visitor with norm 2 (white in $m_{S}$ ) than there are $\mathrm{B}$ residents with norm 1 (grey in $1-m_{B}$ ) for every $\mathrm{S}$ visitor with norm 1 (grey in $m_{S}$ ). Because assortment $(a)$ is high, miscoordination will be low, and average payoffs to individuals with norm 1 and norm 2 will be heavily influenced by how many inter-ethnic (versus intra-ethnic) coordinations they engaged in, thereby earning the high marginal inter-ethnic coordination payoff $g_{S}$. Thus, S individuals with norm 2 have higher average payoffs than those with norm 1 . Norm 1 will decrease in frequency in group S, while norm 2 increases.

From the perspective of the other group, B visitors and residents with norm 1 have more opportunities to engage in inter-ethnic coordination with $\mathrm{S}$ individuals who have norm 1 than $\mathrm{B}$ visitors and residents with norm 2 have to engage in inter-ethnic coordination with $\mathrm{S}$ individuals who have norm 2 . This can be seen in the interaction associations in Fig 1 . In $R_{S}$ there are more $\mathrm{S}$ residents with norm 1 (grey in $1-m_{S}$ ) for every B visitor with norm 1 (grey in $m_{B}$ ) than there are $\mathrm{S}$ residents with norm 2 (white in $1-m_{S}$ ) for every B visitor with norm 2 (white in $m_{B}$ ). Similarly, in $V_{S}$ there are more $\mathrm{S}$ visitors with norm 1 (grey in $m_{S}$ ) for every B resident with norm 1 (grey in $1-m_{B}$ ) than there are $\mathrm{S}$ visitors with norm 2 (white in $m_{S}$ ) for every $\mathrm{B}$ resident with norm 2 (white in $1-m_{B}$ ). As above, average payoffs to individuals with norm 1 and norm 2 will be heavily influenced by how many inter-ethnic (versus intra-ethnic) coordinations they engaged in, thereby earning the moderate marginal inter-ethnic coordination payoff $g_{B}$. Thus, B individuals with norm 1 have higher average payoffs than those with norm 2 . Norm 2 will decrease in frequency in group $\mathrm{B}$, while norm 1 increases.

To review, due to their higher average probability of inter-ethnic coordination yielding a payoff bonus ( $g_{k}$ for group $k$ ), individuals with norm 2 initially increase in frequency in minority group $\mathrm{S}$, and individuals with norm 1 initially increase in frequency in majority group B. This can be seen in the early stages of the norm frequency trajectories in Fig 3A. The frequency of norm $1\left(p_{1}\right)$ initially decreases in group S and increases in group B. The long-run outcome of the model is determined by the relative rates of these frequency changes in the two groups. If assortment $a$ is high (i.e., the risk of miscoordination is low) and relative group size is held constant (majority group B is always twice the size of minority group S), these rates will be heavily influenced by the relative sizes of the marginal inter-ethnic coordination payoffs. When such a payoff is higher for the minority group than the majority group $\left(g_{S}>g_{B}\right)$, the rate at which the frequency of norm 2 increases in group $\mathrm{S}$ is faster than the rate at which the frequency of norm 1 increases in group B. The faster rate has a powerful influence on the slower rate. 


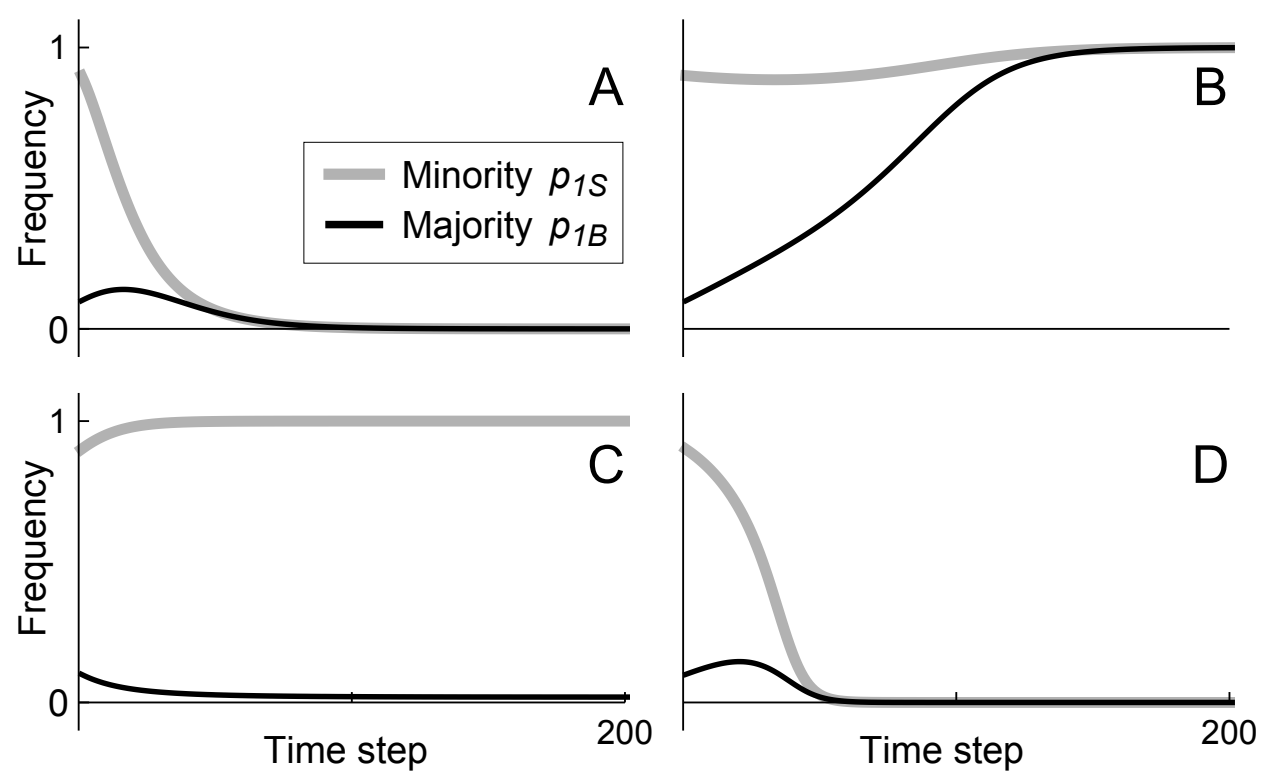

FiguRE 3. Trajectories of the frequency of norm 1 in minority group $\mathrm{S}\left(p_{1 S}\right)$ and in majority group $\mathrm{B}\left(p_{1 B}\right)$ under a subset of conditions shown in Fig. 2. For all simulations, $d_{S}=0.5, \mu=0.5, g_{B}=0.5$, and norm 1 initially occurs at high frequency (0.9) in group $\mathrm{S}$ and low frequency (0.1) in group B. A) When there is low bi-directional inter-ethnic visiting $\left(m_{S}=m_{B}=0.1\right)$ and $\mathrm{S}$ individuals benefit most from inter-ethnic coordination $\left(g_{S}=0.9>g_{B}=0.5\right)$, S-typical norm 1 will be lost from both groups despite high assortment $(a=0.9)$. This corresponds to the upper right quadrants of the plots in Fig. 2A. B) The simulation is identical to that in A, but now $\mathrm{S}$ individuals benefit least from inter-ethnic interaction $\left(g_{S}=0.1<g_{B}=0.5\right)$, resulting in the fixation of S-typical norm 1 in both groups. This corresponds to the upper left quadrants of the plots in Fig. 2A. C) When visitors are restricted from group B but not from group $\mathrm{S}\left(m_{B}=0\right.$ and $m_{S}=$ 0.1 ) norm 1 can persist in group $S$, and at low frequency in group $\mathrm{B}$, regardless of assortment strength (e.g., here $a=0.7$ ), even if $\mathrm{S}$ individuals benefit most from inter-ethnic coordination $\left(g_{S}=0.9>\right.$ $\left.g_{B}=0.5\right)$. See Fig. 2C. D) The simulation is identical to that in $\mathrm{C}$, but now $\mathrm{S}$ visitors are increased $\left(m_{S}=0.4\right)$, resulting in the loss of norm 1 from the population. This corresponds to the upper right quadrants of plots in Fig. 2D

To see this, consider $R_{S}$ and $V_{S}$ in Fig 1 after the frequency of norm 2 has already increased substantially in group $\mathrm{S}$, i.e., the white regions of $1-m_{S}$ and $m_{S}$ are substantially larger. At the same time, because of a slower rate of increase, the frequency of norm 1 has increased only slightly in group B, i.e., the grey regions of $1-m_{B}$ and $m_{B}$ are nearly unchanged. This serves to decrease the probability that $B$ visitors and residents with norm 1 will engage in inter- rather than intra-ethnic 
interactions, thereby decreasing the probability of attaining $g_{B}$, and decreasing the average payoff advantage of norm 1 over norm 2 in group B. When the frequency of norm 1 in group $\mathrm{S}$ drops low enough (e.g., $1-m_{S}$ and $m_{S}$ are almost completely white), there are no longer many opportunities for B individuals with norm 1 to obtain $g_{B}$, while there are now many opportunities for B individuals with norm 2 to obtain this marginal inter-ethnic coordination payoff. As a result, the average payoffs of norm 2 in the group B overtake those of norm 1, and the frequency of norm 1 begins to decrease, rather than increase, in group B. This can be seen in the humped trajectory for $p_{1 B}$ in Fig $3 \mathrm{~A}$.

Note that, as the frequency of norm 1 decreases in group $\mathrm{S}$, the payoff advantage of norm 1 over norm 2 in group B decreases as a result of fewer opportunities for B individuals with norm 1 to engage in inter- versus intra-ethnic coordination. Similarly, as the frequency of norm 2 decreases in group B, the payoff advantage of norm 2 over norm 1 in the group $\mathrm{S}$ decreases. Thus, the rate of decrease of norm 1 in group $\mathrm{S}$ slows as it becomes less common (negative frequency dependence). This can be seen in the trajectory of $p_{1 S}$ in Fig $3 \mathrm{~A}$. If assortment were perfect $(a=1)$, the rate of decrease of norm 1 in group $\mathrm{S}$ would slow to zero before norm 1 is eliminated, and a stable mixed equilibrium would be attained as explained above in Section 3.1.1 and shown in Fig 4D. However, when occasional miscoordination occurs $(a<1)$, payoffs also exhibit a positive dependence on the frequency of the norm in the population, such that the least common norm has the highest risk of miscoordination, lowering its average payoff. The strength of this positive frequency dependence increases as a rare norm becomes less common in the population (see equation 8 and Section 3.1.2, above). Thus, even though the rate of decrease of the frequency of norm 1 in group $\mathrm{S}$ slows as it becomes less common, norm 1 will eventually be driven to extinction in group S under these conditions of imperfect assortment as a result of the influence of an interacting process of positive frequency dependence. This will occur even if assortment is very high, yet imperfect (Fig 4C).

In summary, in this model, the reason S-typical norms are lost when S individuals benefit most from inter-ethnic coordination is because the few $\mathrm{S}$ individuals who have B-typical norms receive a higher average payoff than their fellow S members, and are thus copied at higher rates. The few B individuals with S-typical norms initially have higher average payoffs than their fellow B members, but the pool of S individuals with whom they can successfully coordinate disappears before their numbers have a chance to grow substantially, and thus they in turn disappear. The eventual result is the complete replacement of the S-typical norm by the B-typical norm in both groups.

\section{D.2. Bi-directional visiting: maintenance of the minority-typical norm.} The minority S-typical norm 1 goes to fixation in the population when assortment $(a)$ is high and B individuals receive a greater benefit than $\mathrm{S}$ individuals from interethnic coordination $\left(g_{B}>g_{S}\right)$. The dynamics are very similar to those described above in Section D.1. As before, B individuals with norm 1 engage in more interethnic coordination, and thus receive a higher average payoff as a result of the large $g_{B}$, than do B individuals with norm 2. Norm 1 thus increases in group B. Similarly, $\mathrm{S}$ individuals with norm 2 engage in more inter-ethnic coordination, and thus receive a higher average payoff as a result of the moderate $g_{S}$, than do $\mathrm{S}$ individuals with norm 2. Norm 2 thus increases in group S. If groups $\mathrm{S}$ and B were the same size, the 
182 simple fact that $g_{B}>g_{S}$ would result in a faster rate of increase for norm 1 in group 183 B than for norm 2 in group $\mathrm{S}$, and norm 2 would go to fixation in both groups via 184 the mechanism described in the previous section. However, in order for this to occur 185 when the groups are unequal in size, the marginal interethnic coordination payoff to 186 group B, $g_{B}$, must be substantially larger than that of group $\mathrm{S}, g_{S}$.

187 To understand why, consider the interaction associations $R_{S}$ and $V_{S}$ in Fig 1 . The 188 probability that $\mathrm{B}$ residents and visitors with norm 1 will engage in inter-ethnic 189 coordination is lower than the probability that $\mathrm{S}$ residents and visitors with norm 2 190 will engage in inter-ethnic coordination. This is due to the fact that the contingent of $191 \mathrm{~S}$ residents is much larger than the contingent of $\mathrm{S}$ visitors in $V_{S}$, while the contingent 192 of $\mathrm{S}$ residents is not substantially larger than than the contingent of $\mathrm{B}$ visitors in $R_{S}$.
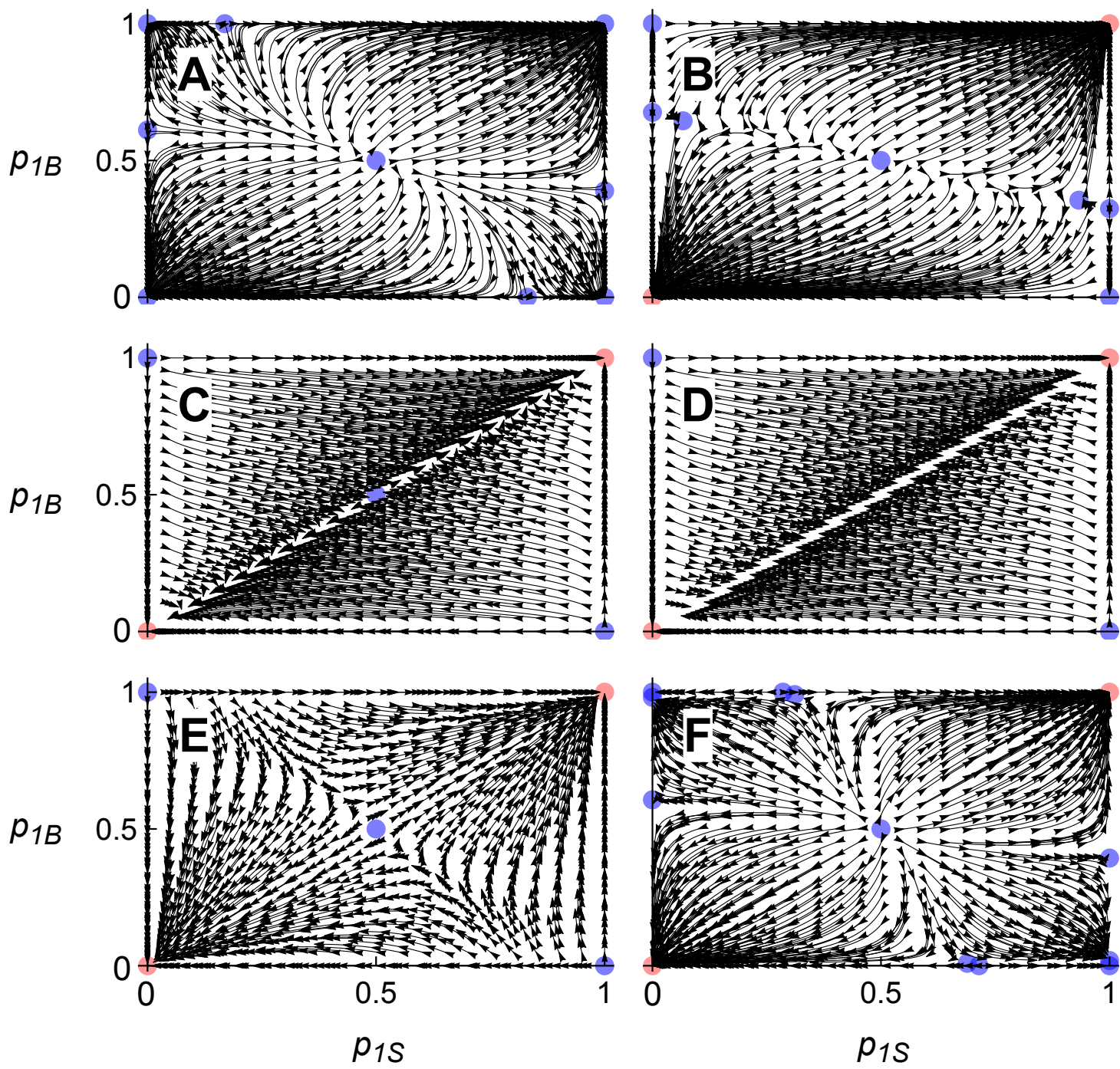

Figure 4. (Caption next page.) 
Figure 4. (Previous page.) Simulated trajectories of the frequencies of norm 1 in minority group $\mathrm{S}\left(p_{1 S}\right)$ and in majority group $\mathrm{B}\left(p_{1 B}\right)$, given a range of initial norm 1 frequencies in each group. For all plots $d_{S}=0.5, \mu=0.5$, and $g_{B}=0.5$. Numerically-solved equilibria are shown in blue. In plots B-E, all of these blue equilibria are unstable. In plots $\mathrm{B}-\mathrm{F}$ the model is undefined at $p_{1 S}=p_{1 B}=0$ (loss of norm 1) and $p_{1 S}=p_{1 B}=1$ (fixation of norm 1). However, these latter two states constitute long-run tendencies of the model and are shown in red. A) When interaction is random with respect to norm $(a=0)$, there is low bi-directional inter-ethnic visiting $\left(m_{S}=m_{B}=0.1\right)$, and $\mathrm{S}$ individuals benefits most from inter-ethnic coordination $\left(g_{S}=0.9>\right.$ $\left.g_{B}=0.5\right)$, positive frequency-dependent selection drives the initially most common norm in each group to fixation. Only corner equilibria are stable. B) Conditions are identical to those in A, but assortment is moderate $(a=0.5)$, resulting in either the loss or fixation of norm 1 in both groups simultaneously. C) Conditions are identical to those in $\mathrm{A}$, but assortment is extremely high $(a=0.99)$. As in $\mathrm{B}$, norm 1 either goes to fixation in both groups, or is lost from both groups. D) Conditions are identical to those in A, but assortment is perfect $(a=1)$, resulting in a diagonal line of mixed equilibria, such that $p_{1 S}=p_{1 B}$. E) Assortment is high $(a=0.9)$ and $\mathrm{B}$ individuals benefit most from inter-ethnic coordination $\left(g_{B}=0.5>g_{S}=0.1\right)$. As long as norm 1 is initially common in group $\mathrm{S}$ and present in group $\mathrm{B}$, it will go to fixation in both groups. F) Assortment is moderately high $(a=0.7)$, visitors are restricted from group $B$ but not group $S\left(m_{B}=0\right.$ and $\left.m_{S}=0.1\right)$, and $S$ individuals benefit most from inter-ethnic interaction $\left(g_{S}=0.9>g_{B}=0.5\right)$. The initially most common norm in each group attains high frequency. Under these conditions, the model is undefined for $p_{1 S}=0$ and $p_{1 S}=1$ (loss or fixation of norm 1 in group $\mathrm{S}$ ). Notice the two stable equilibria just below $p_{1 B}=1$ and just above $p_{1 B}=0$ as $p_{1} S$ approaches 0 and 1 , respectively, demonstrating that the S-typical norm can persist at low frequency in group B at equilibrium (also seen in Fig 3C).

Thus, in order for the rate of increase of norm 1 in group B to surpass the rate of increase of norm 2 in group $\mathrm{S}$, the marginal inter-ethnic coordination payoff bonus to B individuals, $g_{B}$, must be sufficiently larger than that of $\mathrm{S}$ individuals, $g_{S}$, in order to overcome the lower probability that B individuals will engage in inter-ethnic coordination. Norm frequency trajectories under these conditions are shown in Fig $3 \mathrm{~B}$ and Fig $4 \mathrm{E}$.

In summary, in this model, the reason S-typical norms go to fixation when B individuals benefit most from inter-ethnic coordination is because the few $\mathrm{B}$ individuals who have S-typical norms receive a higher average payoff than their fellow B members, and are thus copied at higher rates. The few $\mathrm{S}$ individuals with B-typical norms initially have higher average payoffs than their fellow S members, but the pool of B 
individuals with whom they can successfully coordinate disappears before their numbers have a chance to grow substantially, and thus they in turn disappear. For this to occur, B individuals must benefit considerably more from inter-ethnic coordination than do $\mathrm{S}$ individuals in order to overcome the fact that a smaller proportion of $\mathrm{B}$ individuals have the chance to interact with $\mathrm{S}$ individuals than vice versa. Under these conditions, the eventual result is the complete replacement of B-typical norms by S-typical norms in both groups.

D.3. High bi-directional visiting. If the frequency of visiting is increased substantially in both groups, as in Fig 2B, then the dynamics change slightly. To understand why, consider a visiting rate of 0.5 in both groups, meaning that half of each group leaves to visit the other group. In this case, each interaction association in Fig 1 becomes a half-sized, scaled equivalent of the entire population comprising both groups. We can thus consider one interaction association that is the union of the two groups in the top half of Fig 1 . When assortment $(a)$ is low, individuals with the most common norm in the entire population have the lowest risk of miscoordination, and thus the highest average payoff (given marginal inter-ethnic coordination payoffs, $g_{S}$ and $g_{B}$, of the magnitude used in these simulations). B-typical norm 2, being the most common, goes to fixation in the population (lower half of plots in Fig 2B). If assortment is higher, then dynamics depend on the marginal inter-ethnic coordination payoffs. If assortment is extremely high, then, under the initial conditions of the simulations in Fig 2, the probability of inter-ethnic coordination is approximately equal for individuals with each norm in the two groups (i.e., ignore $m_{S}$ and $m_{B}$, such that $\left.p_{1 S} d_{B} p_{1 B} \approx p_{2 S} d_{B} p_{2 B}\right)$. Therefore, if $g_{B}$ is even slightly greater than $g_{S}$, S-typical norm 1 will go to fixation in the population, following the mechanism described above (very top of plots in Fig 2B). As assortment decreases, individuals with the less-common norm 1 suffer greater and greater risk of miscoordination, decreasing their average payoffs. Consequently, in order to overcome this effect so that the frequency of S-typical norm 1 can increase, B individuals must receive an ever-greater marginal inter-ethnic coordination payoff relative to that of $\mathrm{S}$ individuals $\left(g_{B}>>g_{S}\right)$ (upper-left quadrants of plots in Fig 2B).

The reason that increasing the proportion of visitors close to 0.5 increases the scope for the fixation of the S-typical norm in the population (upper left quadrants of the plots for majority group 2 in Fig $2 \mathrm{~A}$ and B) is the result of a combination of two effects. As mentioned above, when there are many visitors and assortment is extremely high, the probability of inter-ethnic coordination is approximately equal for individuals with each norm in the two groups, and the S-typical norm 1 will go to fixation if $g_{B}$ is even slightly greater than $g_{S}$. When the proportion of visitors is low, the probability of inter-ethnic assortment is higher for $\mathrm{S}$ individuals. S individuals with norm 2 therefore often receive $g_{S}$ and therefore have high average payoffs. In order to overcome this effect, such that B individuals with norm 1 have even higher average payoffs, $g_{B}$ must be substantially greater than $g_{S}$.

When there are few visitors and assortment is moderate (e.g., $a$ slightly greater than 0.5 ), the ratio of norm 1 to norm 2 in the interaction association $V_{S}$ is very low, and individuals with norm 1 run a high risk of miscoordination. In $R_{S}$, individuals with norm 1 outnumber those with norm 2 by a moderate amount, and so the risk of miscoordination for norm 1 individuals is lower. However, accounting for both 
interaction associations, the overall risk of miscoordination by individuals with norm 1 is still high, and so average payoffs to norm 1 individuals will be lower. In order for norm 1 to go to fixation in both groups, assortment must increase in order to lower the risk of miscoordination. However, when there are many visitors and assortment is moderate, the ratio of norm 1 to norm 2 in the interaction associations (approximately, the union of the two groups in the top half of Fig 1) is not as low, so the risk of miscoordination by norm 1 individuals is not as high, serving to increase the average payoff of such individuals. Thus, for given values of $g_{S}$ and $g_{B}$, norm 1 can go to fixation in the population at lower levels of assortment when there are many visitors, compared to when there are few visitors. These two process serve to convert the square region of norm 1 fixation in group B in the upper-left quadrant of Fig $2 \mathrm{~A}$ to the larger triangular region in Fig 2B.

In summary, in this model, the reason increasing the number of visitors increases the scope for fixation of the S-typical norm in the population is because, at high levels of assortment, a high proportion of visitors (up to 0.5) decreases the difference in the probabilities that individuals with each norm will engage in inter-ethnic coordination. This allows for fixation of the S-typical norm given smaller differences between marginal inter-ethnic coordination payoffs, $g_{S}$ and $g_{B}$. At moderate levels of assortment, a high proportion of visitors (up to 0.5) decreases the risk of miscoordination for individuals with the S-typical norm by increasing the ratio of individuals with the S-typical norm to those with the B-typical norm during the interaction stage. This serves to increase average payoffs to individuals with the S-typical norm, and allows for the fixation of this norm in the population under lower levels of assortment.

\section{D.4. One-directional visiting: maintenance of the minority norm. When} there are visitors from group $\mathrm{S}$ to group B but not vice versa, the S-typical norm can go to fixation in group B. To understand why, consider Fig 1 when $m_{B}=0$. Initially, most $\mathrm{S}$ visitors to group $\mathrm{B}\left(m_{S}\right)$ have S-typical norm 1. If people assort on norm $(a>0)$ and there is an added benefit to inter-ethnic coordination $\left(g_{S}>0\right)$, the few visitors with B-typical norm 2 gain the highest mean payoff because they are most likely to engage in inter-ethnic coordination with B residents, most of whom share their norm. Meanwhile, among S residents $\left(1-m_{S}\right)$, all of whom engage only in intraethnic interaction, individuals with norm 2 receive a lower average payoff because they are rare (assuming imperfect assortment, $a<1$ ). When $\mathrm{S}$ visitors return home at the conclusion of the interaction phase, they re-integrate with the resident population, and the mean payoff advantage of visitors with norm 2 is diluted.

In the other group, if assortment is strong enough (large $a$ ) and there are enough $\mathrm{S}$ visitors, $\mathrm{B}$ residents with norm 1 receive an average payoff advantage over $\mathrm{B}$ residents with norm 2 . This is because there are more $\mathrm{S}$ visitors with norm 1 with whom they can coordinate to receive the inter-ethnic coordination payoff $g_{B}$. At the conclusion of the interaction phase, there is no reintegration of visitors into group B (i.e., there are no $\mathrm{B}$ visitors), and there is therefore no dilution of the average payoff advantage to norm 1. Thus, the rate of increase of S-typical norm 1 in group B is higher than the corresponding rate for norm 2 in group $\mathrm{S}$. This eventually results in S-typical norm 1 going to fixation in both groups via the mechanism described above in Section D.1 (see top region of plots in Fig 2C). 
However, if assortment is not sufficiently high and/or there are not enough $\mathrm{S}$ visitors, norm 1 individuals in group B do not attain a high enough mean payoff to compensate for the higher risk of miscoordination due to their low frequency in group B. Under these conditions, the initially-most common norm in each group attains high frequency in each group (Fig $4 \mathrm{~F}$ ). If there are relatively few visitors, and assortment on norm is not too low, the S-typical norm 1 can be maintained at low frequency in group B. This is because, at very low frequency, B individuals with norm 1 have a high probability of inter-ethnic coordination with $\mathrm{S}$ visitors, all of whom have norm 1. These payoffs balance the risk of miscoordination with other people in group B. This effect can be seen in the small, but non-zero, equilibrium frequency for norm 1 in group B in Fig 3C.

In summary, the reason why restricting majority, but not minority, visitors can preserve minority-typical norms despite interaction with a more powerful majority group is that it slows the rate at which the majority-typical norm increases in the minority group. This occurs because minority residents with majority-typical norms do relatively poorly in interactions within their own group, where their norms are uncommon and they have no visitors from the majority group with whom to engage in inter-ethnic coordination. The few minority visitors with majority-typical norms achieve higher inter-ethnic coordination payoffs but can do little to offset this effect, as their payoffs are diluted into the average payoff for the majority-typical norm upon their return home (though see caption for Fig 2C). When assortment on norms is low, such minority group individuals also suffer higher average rates of miscoordination in intra-ethnic interactions and their norms are lost from the minority group regardless of the norm frequency in the majority group. When assortment on norms is high, majority group individuals with minority-typical norms receive high average payoffs as a result of inter-ethnic coordination, and they increase quickly in the majority group. Soon, minority visitors with majority-typical norms find few people to coordinate with, further decreasing their average payoff. Consequently, the minority-typical norm goes to fixation in both groups. In this way, a territory with a boundary porous to minority, but not majority, members can function to preserve minority-typical norms despite continued interaction with a powerful majority.

D.5. High one-directional visiting. When there are many $\mathrm{S}$ visitors and assortment $(a)$ is high, $\mathrm{S}$ visitors with B-typical norm 2 receive a high average payoff as a result of their increased chance of inter-ethnic coordination relative to $S$ visitors with norm 1. Because there are more S visitors, there are fewer residents left in group S, where (as long as assortment is not perfect) residents with the more common norm 1 have higher average payoffs than those with norm 2, as described above. When $\mathrm{S}$ visitors return home and reintegrate with residents, the high average payoff of visitors with norm 2 is diluted by the lower average payoff of residents with norm 2 , as described above. Because there are fewer $\mathrm{S}$ residents, there is less dilution of payoffs. However, as long as the marginal inter-ethnic coordination payoff to $\mathrm{S}$ individuals $\left(g_{S}\right)$ is not too large, this effect will still lower the average payoff of $\mathrm{S}$ individuals with norm 2 relative to those with norm 1 . Norm 1 will thus go to fixation in group $\mathrm{S}$. B individuals with S-typical norm 1 receive a high average payoff as a result of the large number of $\mathrm{S}$ visitors with norm 1, and norm 1 will go to fixation in group B as 
long as assortment is high and as long as the frequency of norm 1 in the group $\mathrm{S}$ also remains high (top region of both plots in Fig 2D).

If assortment is relatively high and B individuals benefit most from inter-ethnic interaction $\left(g_{B}>g_{S}\right)$, lowering assortment further decreases the average payoff to $\mathrm{S}$ residents with the uncommon B-typical norm 2. At the same time, lowering $\left(g_{S}\right)$ decreases the average payoff to $\mathrm{S}$ visitors with norm 2 . These two effects lead to the fixation of norm 1 in group S (left side of Group S plot in Fig 2D). In group B, increasing the number of $\mathrm{S}$ residents, especially those with S-typical norm 1, increases the average payoff of $\mathrm{B}$ individuals with norm 1 because they have more opportunities for inter-ethnic coordination. This allows norm 1 to go to fixation in group B at even lower levels of assortment $(a)$. Eventually, if assortment gets too low, then the risk of miscoordination by the initially-uncommon $\mathrm{B}$ individuals with norm 1 overshadows the inter-ethnic coordination payoff and norm 1 is lost from group B (compare left sides of majority plots in Fig $2 \mathrm{C}$ and D).

If there is relatively low assortment and $\mathrm{S}$ individuals benefit most from interethnic interaction $\left(g_{S}>g_{B}\right)$, then increasing the number of $\mathrm{S}$ visitors means less dilution of the payoff advantage accruing to $\mathrm{S}$ visitors with B-typical norm 2 (as described above). A large $g_{S}$ means that $\mathrm{S}$ visitors with norm 2 receive even higher average payoffs. Meanwhile, $\mathrm{S}$ visitors with norm 1 receive a lower average payoff than their fellow visitors because there are fewer B individuals with norm 1 for them to coordinate with. Furthermore, because assortment is lower, they run a higher risk of miscoordination because their norm is uncommon in group B. When the large number of $\mathrm{S}$ visitors returns home, there is less dilution of the average payoff advantage of $\mathrm{S}$ visitors with norm 2 over visitors with norm 1, because there are fewer $\mathrm{S}$ residents with whom to reintegrate. As a result, B-typical norm 2 goes to fixation in group S. As norm 1 disappears from the $\mathrm{S}$ visitors to group B, B individuals with norm 1 no longer receive the inter-ethnic coordination bonus $g_{B}$ to compensate for their higher risk of miscoordination due to their initially-low frequency. Consequently, norm 2 also goes to fixation in group B (lower-right regions of plots in Fig 2D). Norm frequency trajectories under these conditions are shown in Fig 3D.

In summary, when majority visitors to the minority group are restricted but there are many minority visitors to the majority group, the majority-typical norm can go to fixation in the minority group if assortment on norm drops and the inter-ethnic coordination benefit to minority individuals increases (e.g., the majority controls more valuable resources). Under these conditions, inter-ethnic interaction becomes very common for minority group individuals, and minority visitors with majority-typical norms do much better than visitors with minority-typical norms. Because there are fewer minority individuals who do not engage in inter-ethnic interaction (residents), these effects are no longer compensated for by the fact that individuals with minoritytypical norms do better during intra-ethnic interaction at home in the minority group.

\section{Appendix E. Assorting On Group as Well as ON NORM}

The model in the main text assumes that individuals have a probability $a$ of preferentially choosing to interact with someone who shares their norm. However, the model assumes that, within an interaction association (e.g., $R_{S}, R_{1 S}, V_{S}$, and $V_{1 S}$ ), individuals interact at random with respect to ethnic (group) affiliation. Here, we 
allow individuals to preferentially interact with members of the other ethnic group with probability $c$ (the probability of negative assortment). In a given interaction association there are two possible scenarios: 1) Individuals from the other group outnumber individuals from your group. If there is perfect negative assortment on group $(c=1)$, then everyone from your group will interact with someone from the other group. 2) Individuals from your group outnumber individuals from the other group. If $c=1$, then a number of individuals from your group (equal to the number of individuals from the other group) will interact with members of the other group, and the rest will interact with other members of your group. To calculate average payoffs during the interaction step, we must account for both of these scenarios. For instance, assume perfect assortment on norm $(a=1)$ and perfect negative assortment on group $(c=1)$. You are a group $\mathrm{S}$ resident with norm 1 in the interaction association $R_{1 S}$. The number of fellow $\mathrm{S}$ residents in this interaction association (expressed as a fraction of the size of group $\mathrm{S}$ ) is $\left(1-m_{S}\right) p_{1 S}$, while the number of group B visitors is $d_{B} m_{B} p_{1 B}$. If $\left(1-m_{S}\right) p_{1 S} \leq d_{B} m_{B} p_{1 B}$, then everyone in group $\mathrm{S}$ receives the inter-ethnic coordination payoff $1+g_{S}$. However, if $\left(1-m_{S}\right) p_{1 S}>d_{B} m_{B} p_{1 B}$, individuals from group $\mathrm{S}$ receive the inter-ethnic payoff of $1+g_{S}$ with probability $\frac{d_{B} m_{B} p_{1 B}}{\left(1-m_{S}\right) p_{1 S}}$, and the intra-ethnic payoff of 1 with probability $1-\frac{d_{B} m_{B} p_{1 B}}{\left(1-m_{S}\right) p_{1 S}}$. We can define a function for the probability of interacting with a visitor from group B in the interaction association $R_{1 S}$ :

$$
\pi_{V 1}=\min \left\{1, \frac{d_{B} m_{B} p_{1 B}}{\left(1-m_{S}\right) p_{1 S}}\right\}
$$

The average payoff to an S resident with norm 1, given perfect assortment on norm, and perfect negative assortment on group is thus

$$
\left(1+g_{S}\right) \pi_{V 1}+(1)\left(1-\pi_{V 1}\right)
$$

When $\left(1-m_{S}\right) p_{1 S} \leq d_{B} m_{B} p_{1 B}, \pi_{V 1}=1$ and the average payoff is $1+g_{S}$. However, when $\left(1-m_{S}\right) p_{1 S}>d_{B} m_{B} p_{1 B}$, the average payoff is decreased. Following similar reasoning, we can define another function for the probability that an $\mathrm{S}$ visitor interacts with a $\mathrm{B}$ resident in the interaction association $V_{1 S}$ :

$$
\pi_{R 1}=\min \left\{1, \frac{d_{B}\left(1-m_{B}\right) p_{1 B}}{m_{S} p_{1 S}}\right\}
$$

In the case where individuals interact at random with respect to norm $(a=0)$ but have perfect negative assortment on group $(c=1)$, we define a new function for the probability that an $\mathrm{S}$ resident interacts with a $\mathrm{B}$ visitor in $R_{S}$ :

$$
\pi_{V}=\min \left\{1, \frac{d_{B} m_{B}}{1-m_{S}}\right\}
$$

However, unlike above, successful coordination is no longer guaranteed. Only a fraction $p_{1 B}$ of the $\mathrm{B}$ visitors and a fraction $p_{1 S}$ of other $\mathrm{S}$ residents has norm 1. Thus, the average payoff to an $\mathrm{S}$ resident who has norm 1, given random interaction with respect to norm, and perfect negative assortment on group is:

$$
\left(1+g_{S}\right) \pi_{V} p_{1 B}+(1)\left(1-\pi_{V}\right) p_{1 S}
$$


As above, we define another function for the probability that an $\mathrm{S}$ visitor interacts with a $\mathrm{B}$ resident in $V_{S}$ :

$$
\pi_{R}=\min \left\{1, \frac{d_{B}\left(1-m_{B}\right)}{m_{S}}\right\}
$$

We now write a new expression for the average payoff to an $\mathrm{S}$ individual with norm 1 when there is perfect negative assortment on group:

$$
\begin{gathered}
a\left\{\left(1-m_{S}\right)\left[\left(1+g_{S}\right) \pi_{R 1}+(1)\left(1-\pi_{R 1}\right)\right]+\right. \\
\left.m_{S}\left[\left(1+g_{S}\right) \pi_{V 1}+(1)\left(1-\pi_{V 1}\right)\right]\right\}+ \\
(1-a)\left\{\left(1-m_{S}\right)\left[\left(1+g_{S}\right) \pi_{R} p_{1 B}+(1)\left(1-\pi_{R} p_{1 S}\right)\right]+\right. \\
\left.m_{S}\left[\left(1+g_{S}\right) \pi_{V} p_{1 B}+(1)\left(1-\pi_{V} p_{1 S}\right)\right]\right\}
\end{gathered}
$$

To this payoff expression we introduce $c$, the probability of assorting negatively on group, in order to construct a complete payoff expression to replace equation 5 :

$$
\begin{gathered}
w_{1 S}=c a\left\{\left(1-m_{S}\right)\left[\left(1+g_{S}\right) \pi_{R 1}+(1)\left(1-\pi_{R 1}\right)\right]+\right. \\
\left.m_{S}\left[\left(1+g_{S}\right) \pi_{V 1}+(1)\left(1-\pi_{V 1}\right)\right]\right\}+ \\
c(1-a)\left\{\left(1-m_{S}\right)\left[\left(1+g_{S}\right) \pi_{R} p_{1 B}+(1)\left(1-\pi_{R} p_{1 S}\right)\right]+\right. \\
\left.m_{S}\left[\left(1+g_{S}\right) \pi_{V} p_{1 B}+(1)\left(1-\pi_{V} p_{1 S}\right)\right]\right\}+ \\
(1-c) a\left\{\left(1-m_{S}\right)\left[\frac{p_{1 S}\left(1-m_{S}\right)}{R_{1 S}}(1)+\frac{p_{1 B} d_{B} m_{B}}{R_{1 S}}\left(1+g_{S}\right)\right]+\right. \\
\left.m_{S}\left[\frac{p_{1 S} m_{S}}{V_{1 S}}(1)+\frac{p_{1 B} d_{B}\left(1-m_{B}\right)}{V_{1 S}}\left(1+g_{S}\right)\right]\right\}+ \\
(1-c)(1-a)\left\{\left(1-m_{S}\right)\left[\frac{p_{1 S}\left(1-m_{S}\right)}{R_{S}}(1)+\frac{p_{1 B} d_{B} m_{B}}{R_{S}}\left(1+g_{S}\right)\right]+\right. \\
\left.m_{S}\left[\frac{p_{1 S} m_{1}}{V_{S}}(1)+\frac{p_{1 B} d_{B}\left(1-m_{B}\right)}{V_{S}}\left(1+g_{S}\right)\right]\right\}
\end{gathered}
$$

When $c=0$, interaction within the interaction associations is random with respect to group and equation 28 reduces to equation 5 .

The long-run norm frequencies produced by this model are shown in Fig 5 and the dynamics are illustrated in Fig 6. Note the overall similarities to Figs 2 and 3 , indicating that allowing people to preferentially choose interaction partners from members of the other ethnic group has only minor effects on norm dynamics under the parameter conditions of interest.

In general, whenever $g_{S}>0$ and $g_{B}>0$, inter-ethnic coordination yields a higher payoff than intra-ethnic coordination. Therefore, negative assortment on group raises the average payoffs of everyone in the population, and a propensity for such assortment would be expected to evolve. However, payoffs to all individuals are not raised by the same amount. In particular, negative assortment on group in the interaction associations buffers the frequency dependence of payoffs to individuals with normgroup phenotypes that are outnumbered by individuals from the other group who 
have the same norm. This effect is particularly strong when the frequencies of individuals with the same norm from two different groups are very similar, as may often occur when $\mathrm{S}$ residents interact with $\mathrm{B}$ visitors (e.g., $R_{1 S}$ ).

For instance, with a low number of visitors, high assortment on norm, but no assortment by group, the average payoff to $\mathrm{S}$ residents with a rare norm decreases as such residents increase in frequency relative to $\mathrm{B}$ visitors with that norm (negative frequency dependence). However, with perfect negative assortment on group, the average payoff to residents with a rare norm is constant (and high) as they increase in frequency, up until the point when they surpass the frequency of visitors from the other group who have their norm. This results in a higher rate of increase for that norm in the population, all else being equal (compare Figs 3A and 6A), increasing the scope for its fixation (compare Figs 2A and 5A). Similarly, the average payoff to $\mathrm{S}$ residents with a common norm increases steadily as such residents decrease in frequency. However, under negative assortment on group, as soon as the frequency of these $\mathrm{S}$ residents matches the frequency of $\mathrm{B}$ visitors with that norm, their average payoff becomes constant and high. When the frequency of visitors is high, this occurs quickly, increasing the scope for fixation of their norm in the population (compare Figs $2 \mathrm{~B}$ and $5 \mathrm{~B})$.

When visitors are restricted from majority group B but not from minority group $\mathrm{S}\left(m_{B}=0\right.$ and $\left.m_{S}>0\right)$ and assortment is moderate but not too high, the Stypical norm can persist in group B at higher levels when there is negative assortment on group. This is because B individuals with this norm decrease to a low enough frequency such that most interact exclusively with $\mathrm{S}$ visitors, all of whom share their norm, and so receive a high payoff that balances the miscoordination risk faced by the rest of their number (compare Figs $2 \mathrm{C}$ and $5 \mathrm{C}$ and Figs $3 \mathrm{C}$ and $6 \mathrm{C}$ ). Additionally, with negative assortment on group, $\mathrm{S}$ visitors all receive the same high average payoff regardless of norm. When there is a high frequency of visitors, this serves to increase the scope for fixation of the S-typical norm in both groups as long as there is moderate assortment on norm (compare Figs 2D and 5D).

\section{Appendix F. Biased SELECTION OF VISITORS WITH ONE-DIRECTIONAL VISITING}

Imagine that norm 1 is initially common in minority group $\mathrm{S}$, while norm 2 is initially common in majority group $\mathrm{B}$. There is one-way visiting from group $\mathrm{S}$ to $\mathrm{B}$, such that $m_{S}>0$ and $m_{B}=0$. The model presented in the main text assumes that individuals are chosen at random from group $\mathrm{S}$ to compose the contingent of visitors.

We use the mean of the hypergeometric distribution to model sampling without replacement from group $\mathrm{S}$ into the visitor contingent. The mean number of successes in $n$ draws (without replacement) from a population of size $N$ which contains $K$ successes is

$$
n \frac{K}{N}
$$

To apply this sampling to our model, let $n_{\text {tot }}$ be the total number of individuals in group S. Thus, in Expr 29, $N$ is $n_{t o t}, n$ is the number of visitors from group $\mathrm{S}$ $\left(m_{S} n_{t o t}\right)$, and $K$ is the number of individuals in group $\mathrm{S}$ who have norm $j\left(p_{j S} n_{t o t}\right)$. Substituting these definitions into Expr 29, the mean number of individuals with 
480

481

norm $j$ from group $\mathrm{S}$ selected to be visitors is

$$
m_{S} p_{j S} n_{t o t}
$$

By dropping the $n_{\text {tot }}$ in Expr 30, we have the unbiased joint probability that a given $\mathrm{S}$ individual has norm $j$ and is also a visitor:

$$
m_{S} p_{j S}
$$

We now relax the assumption of unbiased sampling and assume that $\mathrm{S}$ individuals self-select into the visitor contingent when they believe that their norms match those of most individuals in the group B. We define the degree of such self-selection bias as $1-b$, where $0 \leq b \leq 1$, such that $b=0$ is complete bias and $b=1$ is no bias. Thus, when $b=0$, the few $\mathrm{S}$ individuals with norm 2 preferentially self-select into $m_{S}$ because they know that their norm is most common in group B. Now imagine that all $\mathrm{S}$ visitors must travel in a boat in order to reach group $\mathrm{B}$. The boat has a finite number of seats, equal to the size of group $S$ multiplied by $m_{S}$. If there are more $\mathrm{S}$ individuals with norm 2 than there are seats in the boat $\left(p_{2 S} \geq m_{S}\right)$, then, when there is complete bias $(b=0)$, the visitor boat will be completely filled with norm 2 individuals, and some norm 2 individuals will potentially be left as residents in group S. However, if there are fewer minority individuals with norm 2 in group $\mathrm{S}$ than there are seats in the boat $\left(p_{2 S}<m_{S}\right)$, then, when there is complete bias, all $\mathrm{S}$ individuals with norm 2 (and some $\mathrm{S}$ individuals with norm 1) will enter the boat and no individuals with norm 2 will be left as residents in group S. We must account for these two scenarios separately. For simplicity, we assume that only individuals with norm 2 in group $\mathrm{S}$ have the ability to bias their self-selection as visitors.

In the scenario $p_{2 S} \geq m_{S}$, the biased joint mean probability that an $\mathrm{S}$ individual will be a visitor and have norm 2 is

$$
m_{S}\left(p_{2 S}\right)^{b}
$$

Note that when $b=1$, Expr 32 becomes Expr 31 for $j=2$. When $b=0$, Expr 32 is equal to $m_{S}$, in which case all visitors have norm 2 , so the joint probability that an individual is a visitor and has norm 2 is simply the probability that an individual is a visitor.

In the scenario $p_{2 S}<m_{S}$, we must make different substitutions into the hypergeometric mean Expr 29. Imagine the visitor boat has a reserved section where individuals with norm 2 are given priority if they want a seat. The rest of the seats in the visitor boat don't have any such rules. The number of seats in the reserved section is equal to the number of individuals in group $\mathrm{S}$ with norm $2, n_{\text {tot }} p_{2 S}$. If there is complete bias, all the reserved seats will be filled with individuals who have norm 2. However, if there is not complete bias, not all individuals with norm 2 will exercise their right to the reserved seats. Some of the reserved seats will be filled by individuals with norm 1 , and some of the unreserved seats will be filled with individuals who have norm 2. Given incomplete bias $(0<b<1)$, the number of individuals with norm 2 who exercise their right to the reserved seats is equal to $n_{t o t} p_{2 S}\left(p_{2 S}\right)^{b}$.

Given incomplete bias, we must now calculate the number of individuals with norm 2 who take unreserved seats. The number of unreserved seats in the visitor boat is the total number of seats in the boat minus the number of reserved seats, $n_{t o t} m_{S}-n_{t o t} p_{2 S}$. This corresponds to $n$ in Expr 29. The population from which we are drawing visitors 
is now the total population of group $\mathrm{S}$ minus the number of individuals who have already filled the reserved seats in the visitor boat, $n_{t o t}-n_{t o t} p_{2 S}$. This corresponds with $N$ in Expr 29. The remaining number of individuals in group $\mathrm{S}$ with norm 2 (assuming incomplete bias) is the total number of individuals in group $\mathrm{S}$ with norm 2 minus the number of individuals with norm 2 that has already taken the reserved seats, $n_{t o t} p_{2 S}-n_{t o t} p_{2 S}\left(p_{2 S}\right)^{b}$. This corresponds with $K$ in Expr 29.

Adding the mean number of individuals with norm 2 in the reserved and unreserved sections of the visitor boat yields

$$
n_{t o t} p_{2 S}\left(p_{2 S}\right)^{b}+n_{t o t}\left(m_{S}-p_{2 S}\right) \frac{n_{t o t} p_{2 S}\left[1-\left(p_{2 S}\right)^{b}\right]}{n_{t o t}\left(1-p_{2 S}\right)}
$$

Dropping the $n_{t o t}$ and simplifying yields the joint mean probability that an S individual is a visitor and has norm 2 under the current scenario,

$$
p_{2 S}\left[\left(p_{2 S}\right)^{b}+\left(m_{S}-p_{2 S}\right) \frac{1-\left(p_{2 S}\right)^{b}}{1-p_{2 S}}\right]
$$

$$
\begin{array}{r}
m_{S}\left[1-\left(p_{2 S}\right)^{b}\right] \quad \text { for } \quad p_{2 S} \geq m_{S} \\
m_{S}-p_{2 S}\left(p_{2 S}\right)^{b}-p_{2 S}\left(m_{S}-p_{2 S}\right) \frac{1-\left(p_{2 S}\right)^{b}}{1-p_{2 S}} \quad \text { for } \quad p_{2 S}<m_{S}
\end{array}
$$

The joint mean probability that an individual has the alternative norm 1 and is also a visitor is

The joint mean probability that an individual has the alternative norm 1 and is also a resident is

$$
\begin{array}{r}
1-p_{2 S}-m_{S}\left[1-\left(p_{2 S}\right)^{b}\right] \quad \text { for } \quad p_{2 S} \geq m_{S} \\
1-m_{S}-p_{2 S}+p_{2 S}\left(p_{2 S}\right)^{b}+p_{2 S}\left(m_{S}-p_{2 S}\right) \frac{1-\left(p_{2 S}\right)^{b}}{1-p_{2 S}} \quad \text { for } \quad p_{2 S}<m_{S}
\end{array}
$$

Exprs 32 and 34 - 40 can now be substituted into equations 3 - 5 to construct four separate pairs of fitness expressions for individuals with norm 1 in group $\mathrm{S}$, norm 2 in group $\mathrm{S}$, norm 1 in group B, and norm 2 in group B. For instance, label Expr 37 as $v$ fill $_{1 S}$, Expr 38 as vspace $1 S$, Expr 39 as $r f i l l_{1 S}$, and Expr 40 as rspace $_{1 S}$. 
Substituting into equations 3 and 4 yields

$$
\begin{aligned}
R_{1 S}^{\text {fill }} & =\operatorname{rfill}_{1 S}+p_{1 B} d_{B} m_{B} \\
V_{1 S}^{\text {fill }} & =\operatorname{vfill}_{1 S}+p_{1 B} d_{B}\left(1-m_{B}\right) \\
R_{1 S}^{\text {space }} & =\text { rspace }_{1 S}+p_{1 B} d_{B} m_{B} \\
V_{1 S}^{\text {space }} & =\text { vspace }_{1 S}+p_{1 B} d_{B}\left(1-m_{B}\right)
\end{aligned}
$$

Substituting these into the fitness expression, equation 5, yields a pair of fitness expressions under the conditions that minority group $\mathrm{S}$ has a restricted access homeland and $\mathrm{S}$ individuals with norm 2 self-select as visitors with bias $1-b$.

$$
\begin{gathered}
w_{1 S}=a\left\{\left(1-m_{S}\right)\left[\frac{r f i l l_{1 S}}{R_{1 S}^{f i l l}}(1)+\frac{p_{1 B} d_{B} m_{B}}{R_{1 S}^{f i l l}}\left(1+g_{S}\right)\right]+\right. \\
\left.m_{S}\left[\frac{v f i l l_{1 S}}{V_{1 S}^{f i l l}}(1)+\frac{p_{1 B} d_{B}\left(1-m_{B}\right)}{V_{1 S}^{f i l l}}\left(1+g_{S}\right)\right]\right\}+ \\
(1-a)\left\{\left(1-m_{S}\right)\left[\frac{r f i l l_{1 S}}{R_{S}}(1)+\frac{p_{1 B} d_{B} m_{B}}{R_{S}}\left(1+g_{S}\right)\right]+\right. \\
\left.m_{S}\left[\frac{v f i l l_{1 S}}{V_{S}}(1)+\frac{p_{1 B} d_{B}\left(1-m_{B}\right)}{V_{S}}\left(1+g_{S}\right)\right]\right\} \text { for } \quad p_{2 S} \geq m_{S}
\end{gathered}
$$

and

$$
\begin{gathered}
w_{1 S}=a\left\{\left(1-m_{S}\right)\left[\frac{\text { rspace }_{1 S}}{R_{1 S}^{\text {space }}}(1)+\frac{p_{1 B} d_{B} m_{B}}{R_{1 S}^{\text {space }}}\left(1+g_{S}\right)\right]+\right. \\
\left.m_{S}\left[\frac{\text { s space }_{1 S}}{V_{1 S}^{\text {space }}}(1)+\frac{p_{1 B} d_{B}\left(1-m_{B}\right)}{V_{1 S}^{\text {space }}}\left(1+g_{S}\right)\right]\right\}+ \\
(1-a)\left\{\left(1-m_{S}\right)\left[\frac{\text { rspace }_{1 S}}{R_{S}}(1)+\frac{p_{1 B} d_{B} m_{B}}{R_{S}}\left(1+g_{S}\right)\right]+\right. \\
\left.m_{S}\left[\frac{\text { vspace }_{1 S}}{V_{S}}(1)+\frac{p_{1 B} d_{B}\left(1-m_{B}\right)}{V_{S}}\left(1+g_{S}\right)\right]\right\} \text { for } p_{2 S}<m_{S}
\end{gathered}
$$

Note that when there is no bias $(b=1)$, both equations 45 and 46 simplify to equation 5. Analogous fitness expressions can be constructed for $w_{1 B}, w_{2 S}$, and $w_{2 B}$. In this model we eliminate bias ( $\operatorname{set} b=1$ ) as soon as the frequency of norm 2 in group $\mathrm{B}$ is no longer greater than the frequency of norm 2 in group $\mathrm{S}\left(p_{2 B}<p_{2 S}\right)$. This implements our assumption that $\mathrm{S}$ individuals with norm 2 would not preferentially self-select to become visitors if they knew that their norm was not more common in group B than it is in their own group.

We are interested to know whether biasing the selection of visitors on the basis of norms affects the analysis of the model presented in the main text. We explore this with simulations. Fig 7 presents a comparison of simulations using the model in the main text and simulations using the above model with a strong bias $(b=0.1)$ favouring the selection of individuals with norm 2 as $\mathrm{S}$ visitors to group $\mathrm{B}$, where norm 2 is initially common in group B. This analysis suggests that, under conditions 
of one-directional visiting of S individuals to group B, allowing bias in the selection of visitors does not fundamentally change the long-run outcome of the model, but rather increases the number of time steps necessary to reach equilibrium. In particular, when assortment is high and there are relatively few $\mathrm{S}$ visitors, S-typical norm 1 can go to fixation in both groups even if $\mathrm{S}$ individuals benefit most from inter-ethnic coordination and the few $\mathrm{S}$ individuals with norm 2 preferentially self-select to visit group B, where their norms are initially common (Fig 7B).

To understand why biasing visitor selection has only a minor effect on norm dynamics in the model parameter space of interest, consider the effects of such bias on the interactions of $\mathrm{S}$ individuals. In group $\mathrm{S}$, strong visitor selection bias means that nearly all individuals with norm 2 leave to engage in inter-ethnic interaction with group B. Because norm 2 is initially common in group B, a large number of these $\mathrm{S}$ visitors receive the inter-ethnic coordination payoff bonus $\left(g_{S}\right)$. However, if assortment on norm is imperfect $(a<1)$ these visitors also risk miscoordination with the initially-small number of $\mathrm{B}$ residents who have norm 1. In contrast, $\mathrm{S}$ residents, almost all of whom have norm 1 , have no opportunity to receive the inter-ethnic coordination payoff (because there are no B visitors), but, at the same time, they have a very low risk of miscoordination. Under the model conditions of Fig $7 \mathrm{~B}$, the average payoff resulting from guaranteed coordination with co-ethnics outweighs the average payoff of potential inter-ethnic coordination, given a risk of miscoordination. Thus, in group $\mathrm{S}$, individuals with norm 1 have a higher average payoff and increase in frequency.

In group B, residents with norm 2 initially have high average payoffs because, due to the bias, nearly all $\mathrm{S}$ visitors have norm 2, facilitating the inter-ethnic coordination payoff. However, both $\mathrm{B}$ resident and $\mathrm{S}$ visitor individuals with norm 2 risk miscoordination with the small number of individuals in group B who have norm 1 . In contrast, $\mathrm{B}$ residents with norm 1 initially receive low average payoff because there are almost no $\mathrm{S}$ visitors with norm 1, and they are still a minority of the B residents. They therefore suffer high levels of miscoordination and cannot gain the inter-ethnic coordination bonus. However, due to the norm frequency changes in group S, soon $\mathrm{S}$ visitors comprise mostly individuals with norm 1, as individuals with norm 2 disappear from group S. This eventually drives up the average payoff to B individuals with norm 1, and norm 1 goes to fixation in group B as well.

In summary, biasing visitor selection in group $\mathrm{S}$, such that nearly all individuals with norm 2 are sent away as visitors, results in a nearly homogeneous population of residents who benefit from the low risk of miscoordination. Even though many visitors, nearly all of whom have norm 2, come back well-off after engaging in interethnic coordination, a non-trivial number also come back having miscoordinated. On average, higher payoffs are obtained by residents. However, from the perspective of those in group $\mathrm{B}$, biasing the selection of $\mathrm{S}$ visitors means that most individuals in the majority group receive higher payoffs, at least initially.

\section{APPENDIX G. A MODEL WITH NORMS AND MARKERS}

The model in the main text assumes that individuals have an ability $0 \leq a \leq 1$ to choose interaction partners who have a norm matching theirs. This ability is assumed to be unaffected by the frequency of norms in the population. Thus, an 
individual with a very rare norm has the same probability of choosing an interaction partner with a matching norm as does an individual whose norm is very common in the population. This assumption seems unsatisfying. To add more realism into the model, we introduce markers (following McElreath et al. 2003).

Coordination norms can be difficult to detect in other people until they are used in actual coordination (e.g., you might not know that a partner has a norm for haggling until you attempt to barter with her). In the real world, many norms co-occur with easily-identifiable markers (e.g., clothing, ornamentation, language). If covariance between norms and markers is high, markers can be used as reliable signals of who to coordinate with (e.g., people who speak language A tend to be hagglers, while those who speak language B tend to abide by fixed prices). If markers are easily observable, it is plausible that the ability of people to assort on marker is not strongly linked to a marker's frequency in the population. In other words, people with a rare marker should be able to find each other more easily within a larger population of people with an alternative marker.

In this norm-maker model, we assume there are two norms (1 and 2) and two markers (1 and 2). Initially, norm 1 and marker 1 are both common in minority group S, while norm 2 and marker 2 are both common in majority group B. There are four different norm-marker phenotypes, the frequencies of which are designated $x_{11 k}, x_{12 k}, x_{21 k}$, and $x_{22 k}$, corresponding, respectively, to individuals with norm 1 and marker 1, norm 1 and marker 2, norm 2 and marker 1, and norm 2 and marker 2 in group $k$. The frequency of norm 1 in group $k$ is $p_{1 k}$, and the frequency of marker 1 in group $k$ is $q_{1 k}$.

Rather than measuring the probability of assorting on norm, $a$ now measures the probability of assorting on marker (equivalent to $1-e$ in McElreath et al. 2003). We redefine $R_{1 S}$ and $V_{1 S}$ so that they represent the number of individuals in $R_{S}$ and $V_{S}$, respectively, that has marker 1 , expressed as a multiple of the size of group $\mathrm{S}$ :

$$
\begin{aligned}
R_{1 S} & =\left(1-m_{S}\right) q_{1 S}+d_{B} m_{B} q_{1 B} \\
V_{1 S} & =m_{S} q_{1 S}+d_{B}\left(1-m_{B}\right) q_{1 B}
\end{aligned}
$$

The payoff expression for an individual with norm 1 and marker 1 in group $\mathrm{S}$ is

$$
\begin{gathered}
w_{11 S}=a\left\{\left(1-m_{S}\right)\left[\frac{x_{11 S}\left(1-m_{S}\right)}{R_{1 S}}(1)+\frac{x_{11 B} d_{B} m_{B}}{R_{1 S}}\left(1+g_{S}\right)\right]+\right. \\
\left.m_{S}\left[\frac{x_{11 S} m_{S}}{V_{1 S}}(1)+\frac{x_{11 B} d_{B}\left(1-m_{B}\right)}{V_{1 S}}\left(1+g_{S S}\right)\right]\right\}+ \\
(1-a)\left\{\left(1-m_{S}\right)\left[\frac{p_{1 S}\left(1-m_{S}\right)}{R_{S}}(1)+\frac{p_{1 B} d_{B} m_{B}}{R_{S}}\left(1+g_{S}\right)\right]+\right. \\
\left.m_{S}\left[\frac{p_{1 S} m_{S}}{V_{S}}(1)+\frac{p_{1 B} d_{B}\left(1-m_{B}\right)}{V_{S}}\left(1+g_{S}\right)\right]\right\}
\end{gathered}
$$

For the copying step of the model, we assume that there is no norm-marker recombination. In other words, people copy norms and markers together as a unit. Under this assumption we now construct a new norm-copying table (Table 3). Recursions for each of the four norm-marker phenotypes in each group are derived from this table. 
For instance, after the copying step, the new frequency of individuals with norm 1 and marker 1 in group $\mathrm{S}$ is

$$
\begin{array}{r}
x_{11 S}^{\prime}=x_{11 S}^{2}+2 x_{11 S} x_{10 S}\left[0.5+\mu\left(w_{11 S}-w_{10 S}\right)\right]+ \\
2 x_{11 S} x_{01 S}\left[0.5+\mu\left(w_{11 S}-w_{01 S}\right)\right]+ \\
2 x_{11 S} x_{00 S}\left[0.5+\mu\left(w_{11 S}-w_{00 S}\right)\right]
\end{array}
$$

TABLE 3. Norm-copying table for individuals with norm-marker phenotypes in group $\mathrm{S}$

\begin{tabular}{ccccc}
$\operatorname{Pr}($ self, other $)$ & $\operatorname{Pr}\left(x_{11 S}\right)^{\mathrm{a}}$ & $\operatorname{Pr}\left(x_{12 S}\right)$ & $\operatorname{Pr}\left(x_{21 S}\right)$ & $\operatorname{Pr}\left(x_{22 S}\right)$ \\
\hline$x_{11 S} x_{11 S}$ & 1 & 0 & 0 & 0 \\
$x_{11 S} x_{12 S}$ & $0.5+\mu\left(w_{11 S}-w_{12 S}\right)$ & $0.5+\mu\left(w_{12 S}-w_{11 S}\right)$ & 0 & 0 \\
$x_{11 S} x_{21 S}$ & $0.5+\mu\left(w_{11 S}-w_{21 S}\right)$ & 0 & $0.5+\mu\left(w_{21 S}-w_{11 S}\right)$ & 0 \\
$x_{11 S} x_{22 S}$ & $0.5+\mu\left(w_{11 S}-w_{22 S}\right)$ & 0 & 0 & $0.5+\mu\left(w_{22 S}-w_{11 S}\right)$ \\
$x_{12 S} x_{11 S}$ & $0.5+\mu\left(w_{11 S}-w_{12 S}\right)$ & $0.5+\mu\left(w_{12 S}-w_{11 S}\right)$ & 0 & 0 \\
$x_{12 S} x_{12 S}$ & 0 & 1 & 0 & 0 \\
$\cdot$ & & & & \\
$\cdot$ & 0 & 0 & 0 & 1 \\
$\cdot$ & & & 0 & \\
$x_{22 S} x_{22 S}$ & 0 & & 0 & \\
\hline
\end{tabular}

${ }^{a} \operatorname{Pr}\left(\right.$ Self $=x_{11 S}$ after copying $\mid$ current value of Self, current value of Other). Remaining column headings interpreted analogously.

We are interested to know whether the results of the analysis in the main text are affected if we require individuals to assort on markers that covary with norms rather than directly on norms themselves. We explore this with simulations. Fig 8 presents simulations of the norm-marker model under conditions analogous to those in Fig 3 using the model in the main text. Importantly, the simulations in Fig 8 assume that, initially, the distributions of both the norms and the markers in each group are very different. This assumption seems reasonable for a situation of interaction between two distinct ethnic groups. In particular, individuals with both norm 1 and marker 1 are most common in group $\mathrm{S}$, and individuals with both norm 2 and marker 2 are initially most common in group B. The similarity of the long-run behavior of these two models suggests that including markers does not fundamentally change the conclusions of the model in the main text (compare Figs 3 and 8).

To understand why the results of the two models are so similar, note that phenotypes comprising the norm typical of one group and the marker typical of the other group are quickly lost from both groups (e.g., $x_{12 S}$ and $x_{12 B}$ in Fig 8). This is because, when assortment on marker is high (i.e., large $a$ ), such individuals, due to their initial low frequency in both groups, suffer a high degree of miscoordination with other individuals who share their marker but not their norm. Consequently, their average payoffs are low and they quickly copy the norm-marker combinations of the other two phenotypes, e.g., $x_{11 S}$ and $x_{22 B}$. This accounts for the initial sharp rise in the frequency of $x_{11 S}$ in minority group $\mathrm{S}$ in Fig 8. In a population consisting of the remaining two phenotypes $\left(x_{11 S}, x_{22 S}, x_{11 B}\right.$, and $\left.x_{22 B}\right)$, assorting on marker and assorting on norm are functionally equivalent. Thus, the same forces affecting 
the dynamics of the model in the main text affect the dynamics of this model, and account for the similarities of Figs 3 and 8.

\section{APPENDIX H. INTER-ETHNIC ASYMMETRIES IN RESOURCES AND BARGAINING} POWER

In this model, marginal inter-ethnic coordination payoffs to individuals from different groups are usually asymmetric $\left(g_{S} \neq g_{B}\right)$. This results in asymmetrical bargaining power. To illustrate, consider inter-ethnic coordination in the context of exchange. It is often true that members of a powerful majority ethnic group (group B in the model) control goods or resources that members of a minority ethnicity (group S) value highly and cannot obtain from co-ethnics. This often strongly motivates minority individuals to coordinate with majority individuals in order to obtain such resources. From the perspective of a minority individual, an inter-ethnic exchange entailing coordination on norm yields a higher payoff than an intra-ethnic exchange coordination. In the model payoff matrix (Table 1), $g_{S}>0$. It may be that the minority group controls no goods or resources that members of the majority find valuable beyond the ordinary. In that case, from the perspective of a majority individual, an exchange entailing coordination on norm with a minority individual yields the same payoff as an exchange coordination with a co-ethnic. Thus, $g_{B}=0$. In this situation, there is an ethnic asymmetry in inter-ethnic coordination payoffs $\left(g_{S}>g_{B}\right)$, such that the group controlling valuable resources (B) receives the least benefit from a successful inter-ethnic exchange.

Given the above scenario, an individual from majority group B $\left(I_{B}\right)$ has greater bargaining power than an individual from minority group $\mathrm{S}\left(I_{S}\right)$. The reason is that $I_{B}$ would lose less by forgoing this particular inter-ethnic interaction than would $I_{S}$. For example, if $g_{S}>g_{B}=0$, then $I_{B}$ would lose a payoff of 1 by forgoing the interaction, whereas $I_{S}$ would lose a larger payoff of $1+g_{S}$ (see Table 1). We would expect an individual who stands to lose more from a failed interaction to be more risk-averse than someone who stands to lose less (Knight 1992, pg 133). Thus, $I_{B}$ has power over $I_{S}$ because $I_{S}$ is willing to sacrifice more in order to ensure that the coordination interaction does not fail. Put another way, whenever $g_{S}>g_{B}, I_{B}$ wields a credible threat of termination. The greater the difference between $g_{S}$ and $g_{B}$, the greater the degree of that threat.

To counteract this threat, $I_{S}$ can offer more to $I_{B}$ in the context of the exchange in order to increase $g_{B}$. In this way, $I_{B}$ would stand to lose more if she forgoes coordination. $I_{S}$ could increase $g_{B}$ through payment in a different currency than that of the original exchange (e.g., through favors, deference, acceptance of disrespect). It may often be the case that $I_{S}$ does not have sufficient control over resources subjectively valuable to $I_{B}$ in order to bring $g_{B}$ all the way up to the level of $g_{S}$. In this case, it will still be in the best interest of both parties to engage in inter-ethnic, as opposed to intra-ethnic, coordination. However, individuals from group B will retain power over individuals from group $\mathrm{S}$, because they would still lose less from termination. Although not explored in our model, note that such power asymmetries may be modified if individuals have choice in, and differential access to, coordination partners (Bowles and Gintis 1993, Bunce and McElreath 2017). 
Thus, ethnic asymmetries in the benefits to inter-ethnic coordination in the payoff structure of this model are a representation of ethnic asymmetries in resource control, and, consequently, in bargaining power. Powerful individuals can exercise their power in order to influence the norms used by weaker individuals during coordination (Knight 1992, Ensminger and Knight 1997, Bunce and McElreath 2017), or they can use it to influence the behaviour of weaker individuals in contexts outside of the original coordination interaction. For simplicity, in this model we have assumed that power differentials generated through coordination interactions do not affect the norms employed in the coordination interactions themselves. Thus, here our definition of bargaining power is broader than that which we have used previously (Bunce and McElreath 2017, following Knight 1992). We now define bargaining power as the degree of any credible threat of terminating an interaction, regardless of whether this threat facilitates the acquiescence to norms on the part of the less-powerful (as in Knight 1992), or their acquiescence to some other behaviour that is not in their subjective interest. 

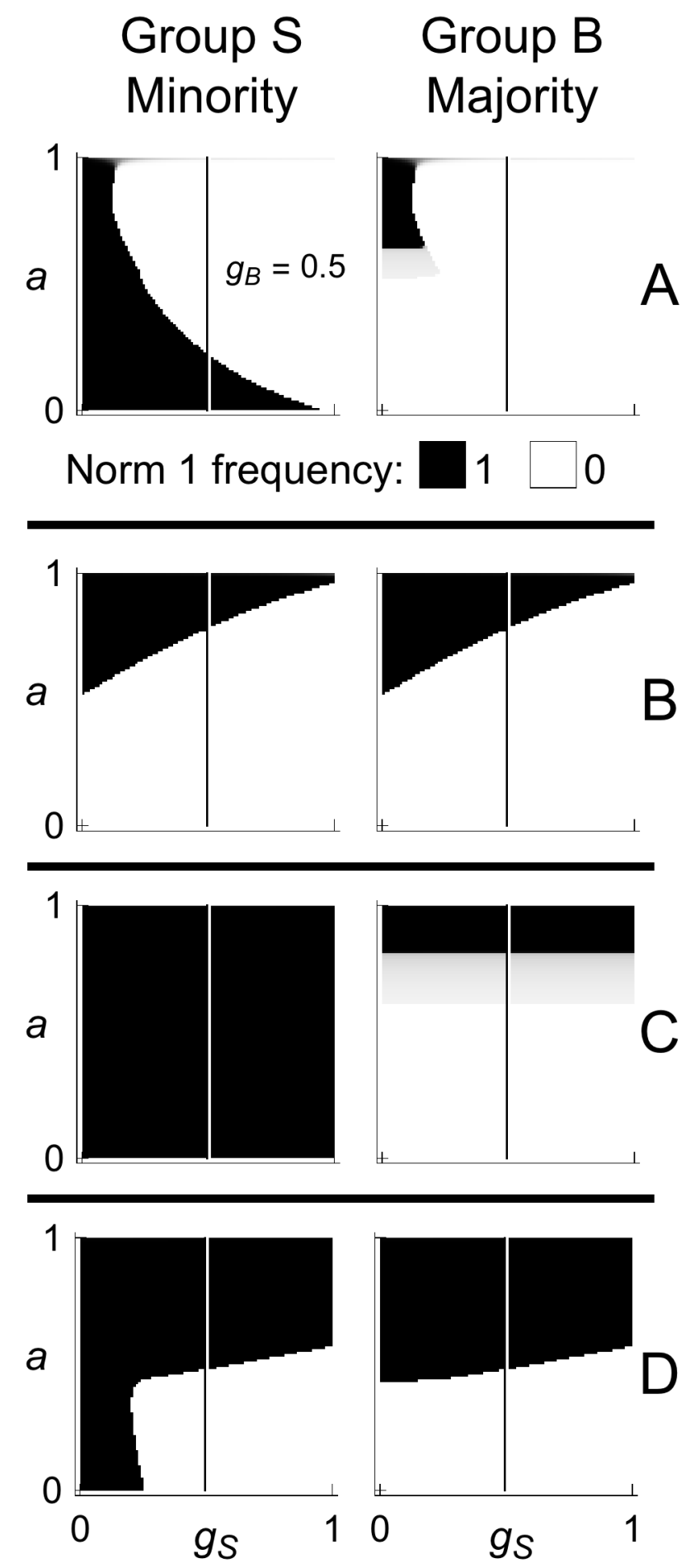

FiguRE 5. Model parameter values identical to Fig 2, but here there is perfect negative assortment on group $(c=1)$. 

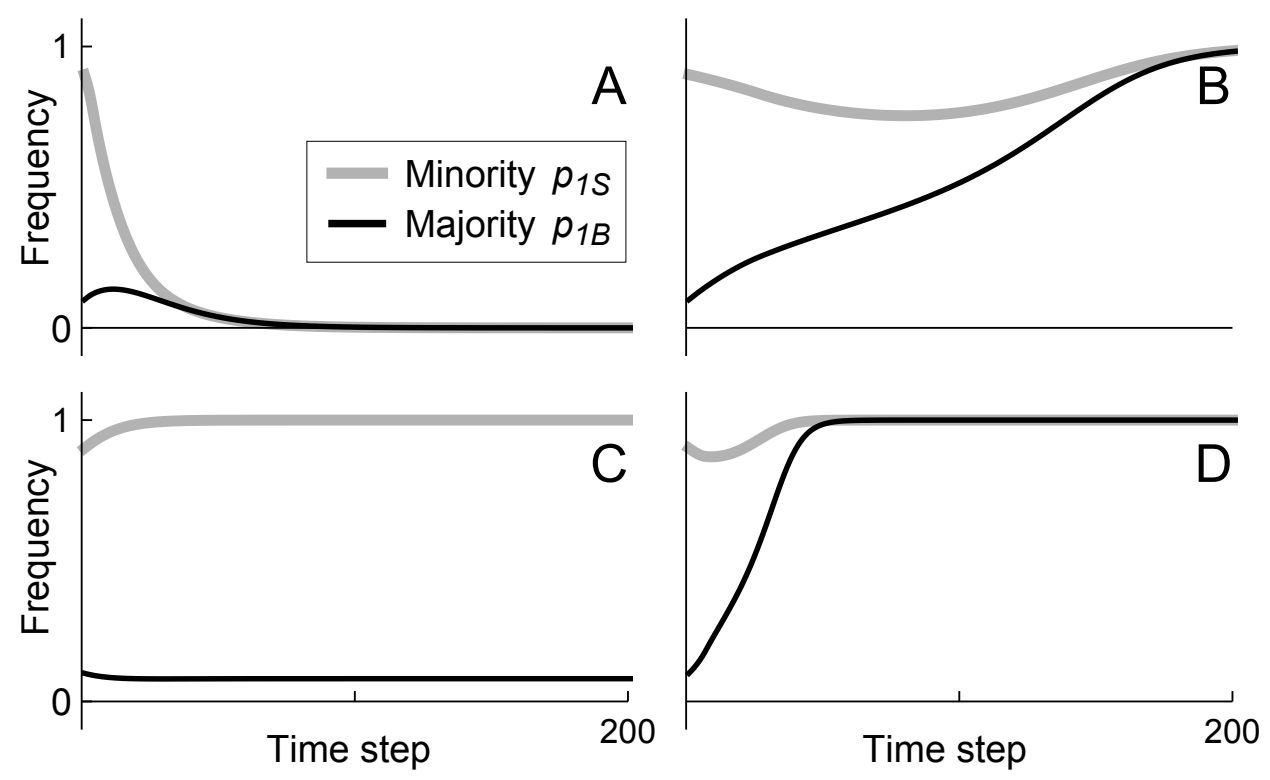

Figure 6. Model parameter conditions identical to Fig 3, but here there is perfect negative assortment on group $(c=1)$. 

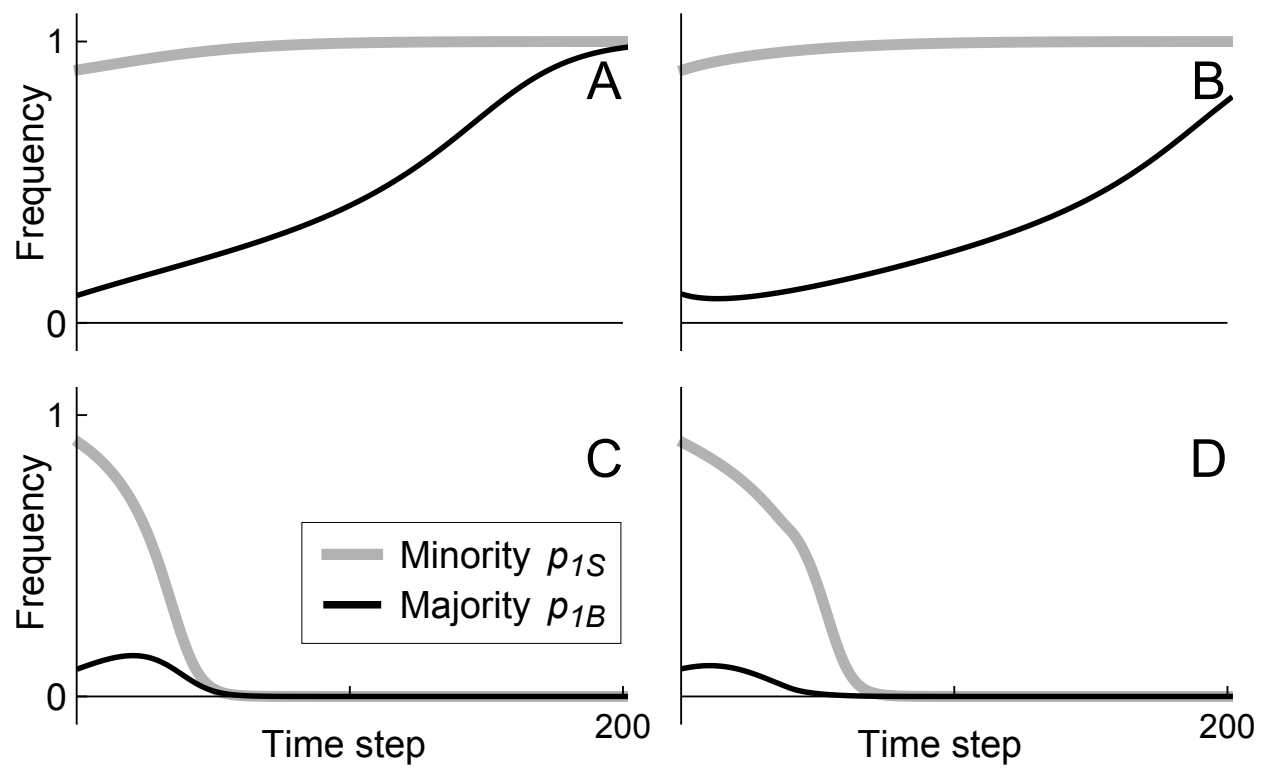

Figure 7. A comparison of models with unbiased and biased onedirectional visiting by $\mathrm{S}$ individuals to group $\mathrm{B}\left(m_{S}>0\right.$ and $\left.m_{B}=0\right)$. For all simulations, $d_{S}=0.5, \mu=0.5, g_{B}=0.5$, and norm 1 initially occurs at high frequency (0.9) in group $\mathrm{S}$ and low frequency $(0.1)$ in group B. A) Assortment is high $(a=0.9)$, there are few $\mathrm{S}$ visitors $\left(m_{S}=0.1\right)$, all of which are chosen at random from group $\mathrm{S}(b=1$, no bias), and $\mathrm{S}$ individuals benefit most from inter-ethnic interaction $\left(g_{S}=0.9>g_{B}=0.5\right)$. S-typical norm 1 goes to fixation in both groups. See Fig. 2C. B) The same as A, but now $\mathrm{S}$ visitors are selected such that individuals with B-typical norm 2 are favored to visit group B $(b=0.1$, strong bias). Note that such bias has little effect on the longrun dynamics. C) Same as A, but now assortment is lower $(a=0.7)$, and there are more $\mathrm{S}$ visitors $\left(m_{S}=0.4\right)$. Norm 1 is quickly lost from the population. See Fig. 2D. D) Same as C, but now S visitors are selected such that individuals with norm 2 are favored $(b=0.1$, strong bias). Note that visitor selection bias has little effect on longrun dynamics under these parameter conditions. 

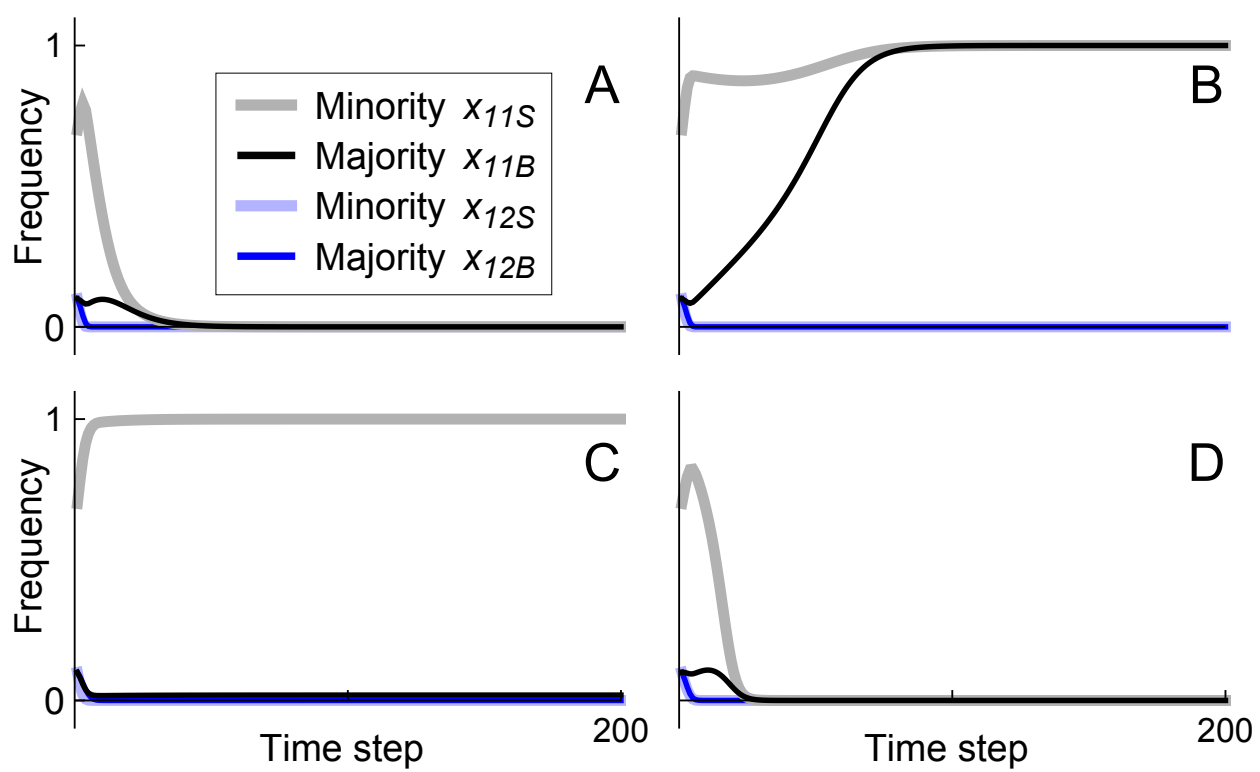

FiguRE 8. A model in which each individual has both a norm (1 or 2 ) and a marker (1 or 2). For all simulations, $d_{S}=0.5, \mu=0.5$, $g_{B}=0.5$, individuals with norm 1 and marker $1\left(x_{11 S}\right)$ initially occur at high frequency $(0.7)$ in minority group $\mathrm{S}$, while all other norm-marker phenotypes occur at low frequency $\left(x_{12 S}=x_{21 S}=x_{22 S}=0.1\right)$, and individuals with norm 2 and marker $2\left(x_{22 S}\right.$ initially occur at high frequency (0.7) in majority group $\mathrm{B}$, while all other phenotypes $\left(x_{11 B}=\right.$ $\left.x_{12 B}=x_{21 B}=0.1\right)$ occur at low frequency. A) Assortment is high $(a=0.9)$, bi-directional inter-ethnic visiting is low $\left(m_{S}=m_{B}=0.1\right)$, and $\mathrm{S}$ individuals benefit most from inter-ethnic coordination $\left(g_{S}=\right.$ $\left.0.9>g_{B}=0.5\right)$. Norm 1 is lost from both groups. This is similar to Fig. 3A. B) The same as A, but now $\mathrm{S}$ individuals benefit least from inter-ethnic interaction $\left(g_{S}=0.1<g_{B}=0.5\right)$, resulting in fixation of norm 1 in both groups. This is similar to Fig. 3B. C) There is one-directional visiting from group $\mathrm{S}$ to group $\mathrm{B}\left(m_{S}=0.1, m_{B}=0\right)$. Norm 1 can persist in group $\mathrm{S}$ despite moderate assortment $(a=0.7)$, even when $\mathrm{S}$ individuals benefit most from inter-ethnic coordination $\left(g_{S}=0.9>g_{B}=0.5\right)$. This is similar to Fig. 3C. D) The same as C, but now $\mathrm{S}$ visitors are increased $\left(m_{S}=0.4\right)$. Norm 1 is lost from both groups. This is similar to Fig. 3D. 


\section{REFERENCES}

Aikman, S. (2003). La Educación Indígena en Sudamérica: Interculturalidad y Bilingüismo en Madre de Dios, Perú. IEP Ediciones, Lima.

Baer, G. (2004). ¿Comes carne de armadillo? acerca del problema de la identidad de los Matsiguenka del Bajo Urubamba en el Oriente Peruano. Boletín de Antropología (Universidad de Antioquia), 18(35):317-336.

Barth, F. (1998). Introduction. In Barth, F., editor, Ethnic Groups and Boundaries: The Social Organization of Culture Difference, pages 9-38. Waveland Press, Inc., Long Grove.

Bicchieri, C. (2006). The Grammar of Society: The Nature and Dynamics of Social Norms. Cambridge University Press, Cambridge.

Bowles, S. and Gintis, H. (1993). The revenge of Homo economicus: contested exchange and the revival of political economy. Journal of Economic Perspectives, $7(1): 83-102$.

Boyd, R. and Richerson, P. J. (1987). The evolution of ethnic markers. Cultural Anthropology, 2(1):65-79.

Boyd, R. and Richerson, P. J. (1990). Group selection among alternative evolutionarily stable strategies. Journal of Theoretical Biology, 145(331-342).

Boyd, R. and Richerson, P. J. (2002). Group beneficial norms can spread rapidly in a structured population. Journal of Theoretical Biology, 215:287-296.

Boyd, R. and Richerson, P. J. (2009). Voting with your feet: payoff biased migration and the evolution of group beneficial behavior. Journal of Theoretical Biology, 257:331-339.

Bunce, J. A. and McElreath, R. (2017). Interethnic interaction, strategic bargaining power, and the dynamics of cultural norms: a field study in an Amazonian population. Human Nature, 28(4):434-456.

Ensminger, J. and Knight, J. (1997). Changing social norms: common property, bridewealth, and clan exogamy. Current Anthropology, 38(1):1-24.

Gilpin, W., Feldman, M. W., and Aoki, K. (2016). An ecocultural model predicts neanderthal extinction through competition with modern humans. Proceedings of the National Academy of Sciences of the USA, 113(8):2134-2139.

Gluckman, M. (1963). Papers in honor of Melville J. Herskovits: Gossip and scandal. Current Anthropology, 4(3):307-316.

Henrich, J. and Gil-White, F. J. (2001). The evolution of prestige: freely conferred deference as a mechanism for enhancing the benefits of cultural transmission. Evolution and Human Behavior, 22:165-196.

Knight, J. (1992). Institutions and Social Conflict. Cambridge University Press, Cambridge.

Kopenawa, D. and Albert, B. (2013). The Falling Sky: Words of a Yanomami Shaman. The Belknap Press of Harvard University Press, Cambridge.

McElreath, R. and Boyd, R. (2007). Mathematical Models of Social Evolution: A Guide for the Perplexed. University of Chicago Press, Chicago.

McElreath, R., Boyd, R., and Richerson, P. J. (2003). Shared norms and the evolution of ethnic markers. Current Anthropology, 44(1):122-129.

Nagel, J. (1996). American Indian Ethnic Renewal: Red Power and the Resurgence of Identity and Culture. Oxford University Press, New York. 
Nave, A. (2000). Marriage and the maintenance of ethnic group boundaries: the case of Mauritius. Ethnic and Racial Studies, 23(2):329-352.

Pisor, A. C. and Gurven, M. (2016). Risk buffering and resource access shape valuation of out-group strangers. Scientific Reports, 6(30435).

Richerson, P. J. and Boyd, R. (2005). Not by Genes Alone: How Culture Transformed Human Evolution. University of Chicago Press, Chicago.

Ross, R. M. and Atkinson, Q. D. (2016). Folktale transmission in the Arctic provides evidence for high bandwidth social learning among hunter-gatherer groups. Evolution and Human Behavior, 37:47-53.

Sahlins, M. (1994). Cosmologies of capitalism: The trans-Pacific sector of "The World System". In Dirks, N. B., Eley, G., and Ortner, S. B., editors, Culture/Power/History: A Reader in Contemporary Social Theory, book section 13, pages 412-455. Princeton University Press, Princeton.

Solway, J. S. and Lee, R. B. (1990). Foragers, genuine or spurious? situating the Kalahari San in history. Current Anthropology, 31(2):109-146.

Wiessner, P. (2005). Norm enforcement among the Ju/'hoansi Bushmen. Human Nature, 16(2):115-145.

Wolf, E. R. (1982). Europe and the People Without History. University of California Press, Berkeley. 